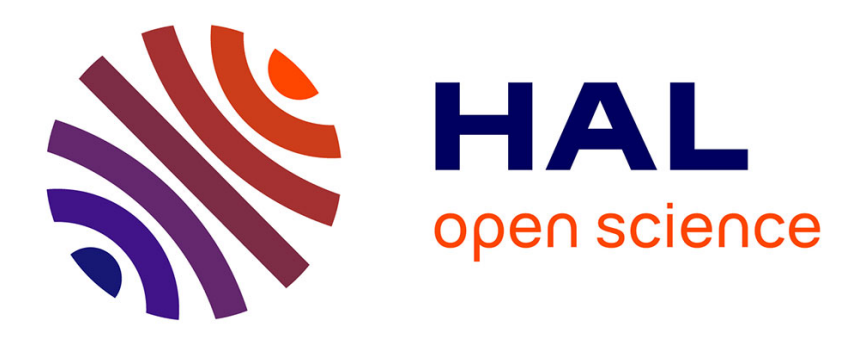

\title{
Large fluctuations in multi-scale modeling for rest hematopoiesis
}

\author{
Céline Bonnet, Sylvie Méléard
}

\section{To cite this version:}

Céline Bonnet, Sylvie Méléard. Large fluctuations in multi-scale modeling for rest hematopoiesis. Journal of Mathematical Biology, 2021. hal-02401831v2

\section{HAL Id: hal-02401831 \\ https://hal.science/hal-02401831v2}

Submitted on 14 Oct 2021

HAL is a multi-disciplinary open access archive for the deposit and dissemination of scientific research documents, whether they are published or not. The documents may come from teaching and research institutions in France or abroad, or from public or private research centers.
L'archive ouverte pluridisciplinaire HAL, est destinée au dépôt et à la diffusion de documents scientifiques de niveau recherche, publiés ou non, émanant des établissements d'enseignement et de recherche français ou étrangers, des laboratoires publics ou privés. 


\title{
Large fluctuations in multi-scale modeling for rest hematopoiesis
}

\author{
Céline Bonnet · Sylvie Méléard
}

Received: date / Accepted: date

\begin{abstract}
Hematopoiesis is a biological phenomenon (process) of production of mature blood cells by cellular differentiation. It is based on amplification steps due to an interplay between renewal and differentiation in the successive cell types from stem cells to mature blood cells. We will study this mechanism with a stochastic point of view to explain unexpected fluctuations on the mature blood cell numbers, as surprisingly observed by biologists and medical doctors in a rest hematopoiesis. We consider three cell types: stem cells, progenitors and mature blood cells. Each cell type is characterized by its own dynamics parameters: its division rate and by the renewal and differentiation probabilities at each division event. We model the global population dynamics by a three-dimensional stochastic decomposable branching process. We show that the amplification mechanism is given by the inverse of the small difference between the differentiation and renewal probabilities. Introducing a parameter $K$ which scales simultaneously the size of the first component, the differentiation and renewal probabilities and the mature blood cell death rate, we describe the asymptotic behavior of the process for large $K$. We show that each cell type has its own size scale and its own time scale. Focusing on the third component, we prove that the mature blood cell population size, conveniently renormalized (in time and size), is expanded in an usual way inducing large fluctuations. The proofs are based on a fine study of the different scales involved in the model and on the use of different convergence and average techniques in the proofs.
\end{abstract}

Keywords Decomposable branching process $\cdot$ Multiscale approximation $\cdot$ Stochastic slow-fast dynamical system $\cdot$ Large fluctuations $\cdot$ Rest Hematopoiesis $\cdot$ Amplification mechanism

C. Bonnet

CMAP, Ecole Polytechnique, CNRS, IP Paris, route de Saclay, 91128 Palaiseau Cedex-France

E-mail: celine.bonnet@polytechnique.edu

S. Méléard

CMAP, Ecole Polytechnique, CNRS, IP Paris, IUF, route de Saclay, 91128 Palaiseau Cedex-France

E-mail: sylvie.meleard@ polytechnique.edu 
Mathematics Subject Classification (2010) MSC 60F05 · MSC 92D25

\section{Introduction}

This paper studies a stochastic model of the process of rest hematopoiesis, the process that produces blood cells in development by differentiation from stem cells to mature blood cells with amplification of the amount of cells. The main goal is to analyze the effects of amplification on the fluctuations of the blood cell number, which observations have shown to be higher than the expected standard fluctuations.

We introduce a multi-type branching process on three types of cells (cf. Gonzalez et al. (2010)-Sect. 12, Axelrod and Kimmel (2002)-Sect. 6.9.1), with a different size scale for each type, and a different time scale for the birth (or death) rate of each of the three types. The dynamics of stem cells (type 1) and progenitors (type 2) result in two distinct events, renewal and differentiation. Each cell of type 1 and 2 divides into two cells at a constant rate, depending on its type. These two new cells are either of the same type as the mother cell (renewal) or of the "next" cell type (differentiation). Mature blood cells (type 3) cells do not divide and can only die at a constant rate. The stem cells are those with the highest capacity for renewal.

In the model, we introduce as unique scaling parameter $K$ the size of the type 1 cell population. The parameter $K$ will also scale the quantities leading to amplification, i.e. the small difference between the differentiation and renewal probabilities at step 2 and the death rate at step 3. We will see (cf. (9)) that the amplification from type 1 cells to type 3 cells is proportional to these two quantities, which will play a main role in our analysis.

The mathematical modeling of hematopoiesis has been firstly introduced in the seminal paper of Till et al. (1964). In this paper, the authors study a binary branching process and show, comparing with biological results, that the probabilistic framework is relevant. Since this pioneering work, many mathematical approaches have been proposed to describe more precisely the cell differentiation kinetics, based either on deterministic or stochastic models (a survey concerning many models can be found in Whichard et al. (2010)). A deterministic approach consists in introducing a dynamical system describing the behavior of the different cell types and in studying different properties of this system, in particular the equilibrium states (see for example Crauste et al. (2008), Loeffler and Wichmann (1980), Marciniak-Czochra et al. (2009), Arino and Kimmel (1986) and the references therein). A noise can also be added to model some random perturbation of these systems, with an eventual delay (see for example Lei and Mackey (2007), Pazdziorek (2014)). Let us note that other stochastic models for hematopoiesis have been introduced (Abkowitz et al. (2000), Roeder and Loeffler (2002), Kimmel and Wazewska-Czyzewska (1982)) but they concentrate on a specific level (either stem cells or red blood cells).

In all this literature, the questions studied by the authors do not concern the impact of the parameters on the amplification. We have only found two papers, Dingli et al. (2007) and Marciniak-Czochra et al. (2009), in which the question was mentioned. They both highlighted the fact that amplification results from the difference between differentiation and renewal probabilities and not from division rates. In our model, 
this difference is calibrated as function of $K$, which allows us to compare the size and time scales of each cell type population sizes: they strongly differ from type 1 to type 3 with increasingly slow time scales and large size scales. Usually, slow and fast components appear naturally, as associated which different species behaviors (see Khammash et al. (2006)), contrary to our case, where the different time scales are deduced from a fine study of the cell differentiation dynamics.

Our approach is inspired by Kang et al. (2014) in which a general theorem for convergence and fluctuations of multiscale processes is obtained but the latter cannot explain the asymptotics of our model. Indeed, in their result, the fluctuations around the deterministic behavior of the slow component are Gaussian, which will not be the case of the type 3 cells dynamics previously described.

In Sect. 2, we introduce the stochastic branching process and the assumptions on the parameters. In Sect. 3, basic martingale properties are stated and some estimates are given for the moments of cell type sizes. We quantify the order of magnitude of the different cell type sizes in function of $K$. On a fixed time interval, we prove the convergence (as $K \rightarrow \infty$ ), of the scaled process to a deterministic degenerate function, only issued from the type 1 dynamics. The two last component dynamics are too slow to be observable at this time scale. In Sect. 4, we study the process on a longer time-scale to capture the asymptotic behavior of type 2 dynamics. We show that the limiting behavior of the two first components process at this time scale is given by an explicit deterministic function $y$. We also show that this time scale is not long enough to observe the very slow dynamics of the third component. Hence, we study the limiting behavior of the process at this new time scale. Then, the second component process goes too fast and doesn't converge in law anymore (in Skorohod topology). We consider the associated occupation measure, as already done in Kurtz (1992). We prove its convergence in a weak sense to the Dirac measure whose support is the unique equilibrium of the second component of the function $y$. Then we deduce the convergence of the third component to a limiting deterministic system involving this equilibrium. In Sect. 5, we study and quantify the fluctuations. We show that the first component behaves at the different time scales as a Brownian motion. This is not the case for the other two ones. Theorem 3 describes the second and third order asymptotics of the second component on its typical time and size scale. The fluctuations around its deterministic limit are not Gaussian. They are described by a finite variation process integrating the randomness of the first component. An independent Brownian motion appears in the third order term. Our main theorem, Theorem 4, describes the fluctuations associated with the third component dynamics. We show that the randomness induced by the dynamics of the two last components is negligible. The fluctuations are due to a cumulative effect of the first component randomness. That's why one observes large and smooth oscillations of the third component.

Notation. $\mathscr{P}(E)$ and $\mathscr{L}(X)$ will denote respectively the space of probability measures on $E$ and the law of a process $X$. As in Kurtz (1992), we will denote by $l_{m}\left(\mathbb{R}_{+}\right)$the space of measures $\mu$ on $[0, \infty) \times \mathbb{R}_{+}$such that $\mu\left([0, t] \times \mathbb{R}_{+}\right)=t$, for each $t \geq 0$. The processes $\langle X\rangle$ and $\langle X, Y\rangle$ will denote respectively the quadratic variation of the process $X$ and the covariation of the processes $X$ and $Y$. 


\section{Model and assumptions}

Cells of type 1 evolve according to a critical linear birth and death process. Birth events correspond to renewal division events, occurring at rate $\frac{\tau_{1}}{2}>0$, while death events correspond to differentiation events occurring at the same rate (a cell of type 1 divides in two cells of type 2). Cells of type 2 divide at rate $\tau_{2}>0$ in two cells of the same type (renewal event) with probability $p_{2}^{R}$ and in two cells of type 3 (differentiation event) with probability $\left.p_{2}^{D}=1-p_{2}^{R} \in\right] 1 / 2,1[$. The mature cells of type 3 die at rate $d_{3}>0$. We can summarize the dynamics as follows. If $N=\left(N_{1}, N_{2}, N_{3}\right)$ denotes the vector of sub-population sizes, the transitions of the hematopoietic process are given by

$$
\begin{aligned}
N_{1} & \longrightarrow N_{1}+1 & \text { at rate }\left(\tau_{1} / 2\right) N_{1} \\
\left(N_{1}, N_{2}\right) & \longrightarrow\left(N_{1}-1, N_{2}+2\right) & \text { at rate }\left(\tau_{1} / 2\right) N_{1} \\
N_{2} & \longrightarrow N_{2}+1 & \text { at rate } \tau_{2} p_{2}^{R} N_{2} \\
\left(N_{2}, N_{3}\right) & \longrightarrow\left(N_{2}-1, N_{3}+2\right) & \text { at rate } \tau_{2} p_{2}^{D} N_{2} \\
N_{3} & \longrightarrow N_{3}-1 & \text { at rate } d_{3} N_{3} .
\end{aligned}
$$

Here, we have assumed that each division is symmetric, so that

$$
p_{2}^{D}+p_{2}^{R}=1 \text {. }
$$

We could have included asymmetric division without changing the results of our study. Indeed it doesn't change the main characteristics of the dynamics.

In the model, we don't consider mortality rates for type 1 and type 2 cells and we assume that cell loss is only due to differentiation in the next cell type. Indeed the hematopoietic stem cell and progenitor death rates, at steady-state, have been biologically estimated and are so small that they can be neglected (cf. Domen et al. (2000)).

As explained in the introduction, we define the scaling parameter $K$ as the size of the type 1 cells population. $K$ is assumed to be large and to scale $p_{2}^{D}-p_{2}^{R}$ and $d_{3}$, in a way which is defined now.

More precisely, we assume that

the size of the type 1 cells population is of order $K$,

and there exists a couple of positive parameters $\left.\left(\gamma_{2}, \gamma_{3}\right) \in\right] 0,1[$ such that

$$
p_{2}^{D}-p_{2}^{R}=K^{-\gamma_{2}} \quad \text { and } \quad d_{3}=\tau_{3} K^{-\gamma_{3}} \quad \text { with } \tau_{3}>0 .
$$

Let us note that (1) and (3) make the probabilities $p_{2}^{R}$ and $p_{2}^{D}$ depend on $K$,

$$
p_{2}^{D}=1-p_{2}^{R}=1 / 2+K^{-\gamma_{2}} / 2 \text {. }
$$

Therefore the dynamics of this cell type is close to a critical process, in the sense that the renewal and differentiation rates are close. 
Assumptions (3) introduce the different time and size scales playing a role for the multi-scale population process describing the dynamics of each cell type size. From now on, since the dynamics depend on $K$, we will denote by $N^{K}$, the population process $N$ previously defined.

We assume in the following that

$$
\gamma_{2}<\gamma_{3}<1
$$

in agreement with biological observations (see ? and Busch et al. (2015)).

Our aim is to finely describe the above dynamics, when $K$ goes to infinity, using appropriate renormalizations.

We will see that a size renormalization is not enough to describe the dynamics of the last two components of the process. A time renormalization is also necessary. More precisely each cell type has its own size scale, of order $K$ for cell type $1, K^{1+\gamma_{2}}$ for cell type 2 (resp. $K^{1+\gamma_{2}+\gamma_{3}}$ for cell type 3 ) and its own time scale, of order 1 for cell type $1, K^{\gamma_{2}}$ for cell type 2 (resp. $K^{\gamma_{3}}$ for cell type 3 ).

The next simulations show the dynamics of the process in the typical time scale of each cell type, namely $K, K^{\gamma_{2}}$ and $K^{\gamma_{3}}$. We visualize the effect of type 1 cells on fluctuations and the typical time and size scale of each cell type. We take as initial condition

$$
N^{K}(0)=(K, 0,0)
$$

and choose $K=2000$ cells of type $1, \gamma_{2}=0.55, \quad \gamma_{3}=0.8$. Hence $K^{\gamma_{2}} \sim 60$ and $K^{\gamma_{3}} \sim 400$.

The others parameters are equal to 1 .

Figure 1 shows the simulation of a trajectory of the process $\left(N^{K}(t), \quad t \in[0, T]\right)$ for $T \sim 1$, decomposed on the three cell types. Figure 2 shows the simulation of a trajectory of the process $\left(N^{K}(t), \quad t \in[0, T]\right)$ for $T \sim K^{\gamma_{2}}$ and Figure 3 shows the simulation of a trajectory of the process $\left(N^{K}(t), \quad t \in[0, T]\right)$ for $T \sim K^{\gamma_{3}}$. The horizontal orange line gives the order of magnitude for size of each cell type ( $K$, resp. $\left.K^{1+\gamma_{2}}, K^{1+\gamma_{2}+\gamma_{3}}\right)$.
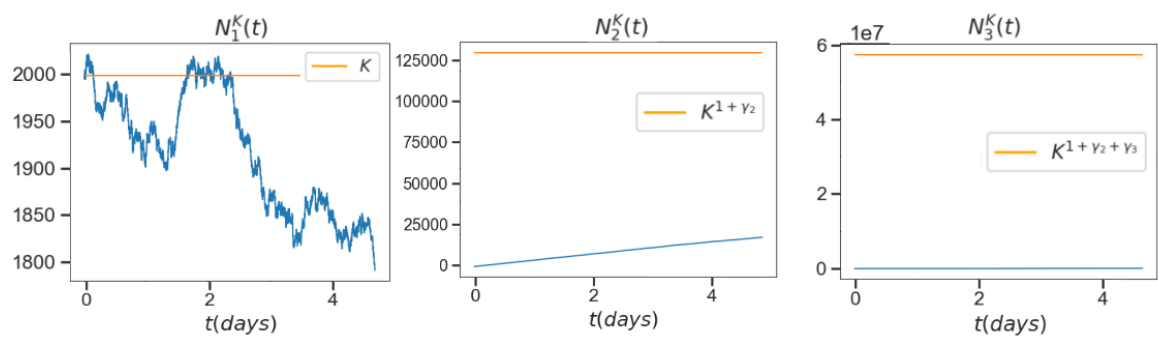

Fig. 1: A trajectory of the $N^{K}$ process for $t \in[0, T]$ with $T=O(1)$ 

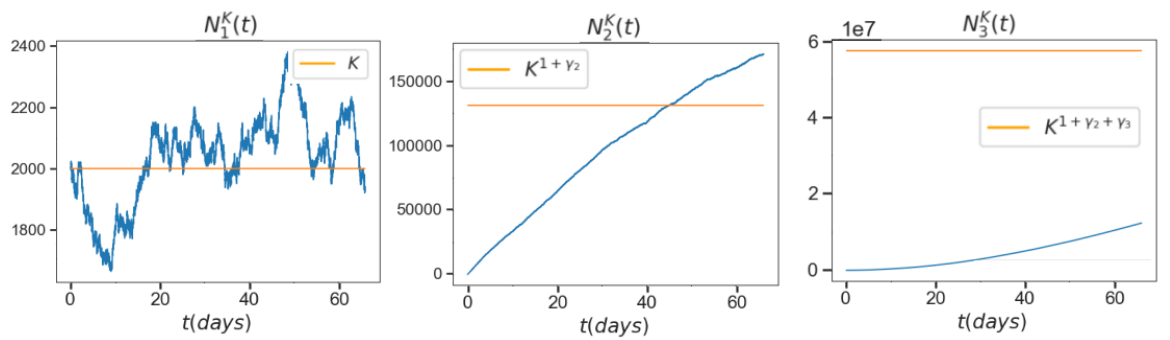

Fig. 2: A trajectory of the $N^{K}$ process for $t \in[0, T]$ with $T=O\left(K^{\gamma_{2}}\right)$
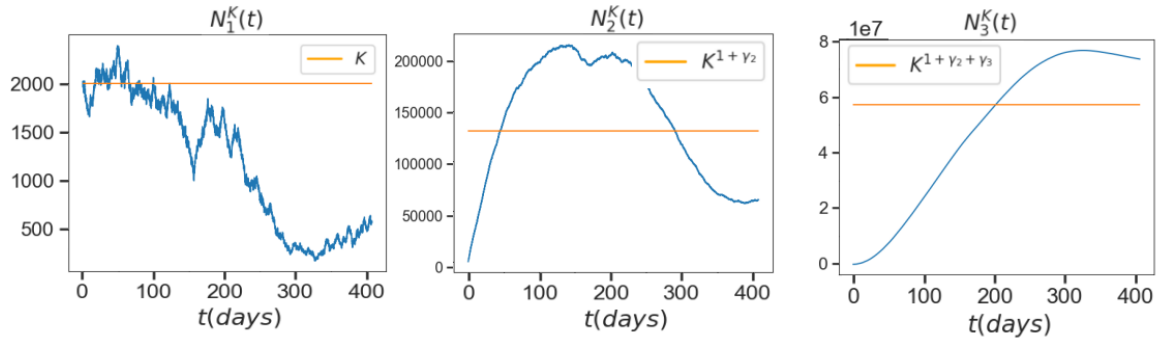

Fig. 3: A trajectory of the $N^{K}$ process for $t \in[0, T]$ with $T=O\left(K^{\gamma_{3}}\right)$

We observe that at a time scale of order 1, the two last components of the process $N^{K}$ are far from their equilibrium size. We observe in Figure 2 that the two first components of $\left(N^{K}(t), \quad t \in[0, T]\right)$ for $T \sim K^{\gamma_{2}}$ evolve around their equilibrium size, which is not the case of the third one. In Figure 3, the process is considered on a longer period of time, $T \sim K^{\gamma_{3}}$ and one sees that the third component hits a neighborhood of its equilibrium. Furthermore, we observe the fluctuations of the components of $N^{K}$ around their equilibrium. We note that they get smoother from cell type 1 to cell type 3 and that the amplitude of the waves get longer.

Our aim is to prove and quantify these different behaviors and to explain these large fluctuations.

\section{The multiscale stochastic process and a first asymptotic result}

3.1 The typical sizes of the stochastic process

Let us now introduce the vector $N^{K}(t)=\left(N_{1}^{K}(t), N_{2}^{K}(t), N_{3}^{K}(t)\right)$ of population sizes at time $t$. The process $N^{K}$ is a decomposable multi-type branching process, that is a Markov jump process whose dynamics is given by the following equations.

We assume that for any fixed $K, N_{1}^{K}(0), N_{2}^{K}(0), N_{3}^{K}(0)$ are integrable. 
Let us denote by $\left(\mathscr{N}_{i}^{j}\right)_{\substack{1 \leq i \leq 3 \\ j \in\{-,+\}}}$ independent Poisson point measures with intensity $d s d u$ on $\mathbb{R}_{+}^{2}$ and introduce the filtration $\left(\mathscr{F}_{t}\right)_{t \geq 0}$ given by

$$
\mathscr{F}_{t}=\sigma\left(\mathscr{N}_{i}^{j}([0, s) \times A) ; i \in\{1, \ldots, 3\}, j \in\{-,+\}, s \leq t, A \in \mathscr{B}\left(\mathbb{R}^{+}\right)\right) .
$$

Then we have

$$
\begin{aligned}
N_{1}^{K}(t)= & N_{1}^{K}(0)+\int_{0}^{t} \int_{\mathbb{R}_{+}} \mathbf{1}_{\mathbf{u} \leq \frac{\tau_{1}}{2} \mathbf{N}_{\mathbf{1}}^{\mathbf{K}}\left(\mathbf{s}^{-}\right)} \mathscr{N}_{\mathbf{1}}^{+}(\mathbf{d s}, \mathbf{d u})-\int_{\mathbf{0}}^{\mathbf{t}} \int_{\mathbb{R}_{+}} \mathbf{1}_{\mathbf{u} \leq \frac{\tau_{1}}{2} \mathbf{N}_{\mathbf{1}}^{\mathbf{K}}\left(\mathbf{s}^{-}\right)} \mathscr{N}_{\mathbf{1}}^{-}(\mathbf{d s}, \mathbf{d u}) \\
N_{2}^{K}(t)=N_{2}^{K}(0) & +2 \int_{0}^{t} \int_{\mathbb{R}_{+}} \mathbf{1}_{\mathbf{u} \leq \frac{\tau_{1}}{2} \mathbf{N}_{\mathbf{1}}^{\mathbf{K}}\left(\mathbf{s}^{-}\right)} \mathscr{N}_{\mathbf{1}}^{-}(\mathbf{d s}, \mathbf{d u})+\int_{\mathbf{0}}^{\mathbf{t}} \int_{\mathbb{R}_{+}} \mathbf{1}_{\mathbf{u} \leq \tau_{2} \mathbf{p}_{2}^{\mathbf{R}} \mathbf{N}_{2}^{\mathbf{K}}\left(\mathbf{s}^{-}\right)} \mathscr{N}_{\mathbf{2}}^{+}(\mathbf{d s}, \mathbf{d u}) \\
& -\int_{0}^{t} \int_{\mathbb{R}_{+}} \mathbf{1}_{\mathbf{u} \leq \tau_{2} \mathbf{p}_{2}^{\mathbf{D}} \mathbf{N}_{2}^{\mathbf{K}}\left(\mathbf{s}^{-}\right)} \mathscr{N}_{\mathbf{2}}^{-}(\mathbf{d s}, \mathbf{d u}) \\
N_{3}^{K}(t)= & N_{3}^{K}(0)+2 \int_{0}^{t} \int_{\mathbb{R}_{+}} \mathbf{1}_{\mathbf{u} \leq \tau_{2} \mathbf{p}_{2}^{\mathbf{D}} \mathbf{N}_{2}^{\mathbf{K}}\left(\mathbf{s}^{-}\right)} \mathscr{N}_{\mathbf{2}}^{-}(\mathbf{d s}, \mathbf{d u})-\int_{\mathbf{0}}^{\mathbf{t}} \int_{\mathbb{R}_{+}} \mathbf{1}_{\mathbf{u} \leq \tau_{3} \mathbf{K}^{-\gamma_{3}} \mathbf{N}_{3}^{\mathbf{K}}\left(\mathbf{s}^{-}\right)} \mathscr{N}_{\mathbf{3}}^{-}(\mathbf{d s}, \mathbf{d u})
\end{aligned}
$$

It can be written for all $t \geq 0$ as,

$$
\begin{aligned}
& N_{1}^{K}(t)=N_{1}^{K}(0)+M_{1}^{K}(t) \\
& N_{2}^{K}(t)=N_{2}^{K}(0)+\tau_{1} \int_{0}^{t} N_{1}^{K}(s) d s-\tau_{2} K^{-\gamma_{2}} \int_{0}^{t} N_{2}^{K}(s) d s+M_{2}^{K}(t) \\
& N_{3}^{K}(t)=N_{3}^{K}(0)+2 \tau_{2} p_{2}^{D} \int_{0}^{t} N_{2}^{K}(s) d s-\tau_{3} K^{-\gamma_{3}} \int_{0}^{t} N_{3}^{K}(s) d s+M_{3}^{K}(t)
\end{aligned}
$$

where $M^{K}=\left(M_{1}^{K}, M_{2}^{K}, M_{3}^{K}\right)$ is a square-integrable martingale such that for all $t \geq 0$,

$$
\begin{aligned}
<M_{1}^{K}>_{t} & =\tau_{1} \int_{0}^{t} N_{1}^{K}(s) d s \\
<M_{2}^{K}>_{t} & =2 \tau_{1} \int_{0}^{t} N_{1}^{K}(s) d s+\tau_{2} \int_{0}^{t} N_{2}^{K}(s) d s \\
<M_{3}^{K}>_{t} & =4 p_{2}^{D} \tau_{2} \int_{0}^{t} N_{2}^{K}(s) d s+\tau_{3} K^{-\gamma_{3}} \int_{0}^{t} N_{3}^{K}(s) d s \\
<M_{1}^{K}, M_{2}^{K}>_{t} & =-\tau_{1} \int_{0}^{t} N_{1}^{K}(s) d s \\
<M_{2}^{K}, M_{3}^{K}>_{t} & =-2 p_{2}^{D} \tau_{2} \int_{0}^{t} N_{2}^{K}(s) d s .
\end{aligned}
$$

Indeed, by standard localization and Gronwall's arguments applied to $\left(N_{1}^{K}(t)\right)_{t}$, we can easily prove that for any $T>0$ and $K \in \mathbb{N}^{*}$,

$$
\mathbb{E}\left[\sup _{t \leq T} N_{1}^{K}(t)\right] \leq\left(2+\mathbb{E}\left[N_{1}^{K}(0)\right]\right) e^{2 \tau_{1} T},
$$

and then that

$$
\mathbb{E}\left[\sup _{t \leq T} N_{2}^{K}(t)\right]<+\infty ; \mathbb{E}\left[\sup _{t \leq T} N_{3}^{K}(t)\right]<+\infty
$$


We obtain from (5) that the function $t \mapsto n(t)=\mathbb{E}\left[N^{K}(t)\right]=\left(n_{1}(t), n_{2}(t), n_{3}(t)\right)$ satisfies the system of equations, for all $t \leq T$,

$$
\left\{\begin{aligned}
n_{1}(t) & =\mathbb{E}\left[N_{1}^{K}(0)\right] \\
\frac{d}{d t} n_{2}(t) & =\tau_{1} n_{1}(t)-\tau_{2} K^{-\gamma_{2}} n_{2}(t) \\
\frac{d}{d t} n_{3}(t) & =2 \tau_{2} p_{2}^{D} n_{2}(t)-\tau_{3} K^{-\gamma_{3}} n_{3}(t) .
\end{aligned}\right.
$$

By assumption (2),

$$
\mathbb{E}\left[N_{1}^{K}(0)\right] \sim K
$$

Therefore there is a unique equilibrium given for all $t \geq 0$ by,

$$
\begin{aligned}
& n_{1}^{*}=\mathbb{E}\left[N_{1}^{K}(0)\right] \sim K \\
& n_{2}^{*}=\frac{\tau_{1} n_{1}^{*}}{\tau_{2}} K^{\gamma_{2}} \sim K^{1+\gamma_{2}} \\
& n_{3}^{*}=\frac{2 p_{2}^{D} \tau_{2} n_{2}^{*}}{\tau_{3}} K^{\gamma_{3}} \sim K^{1+\gamma_{2}+\gamma_{3}} .
\end{aligned}
$$

That suggests to state the following lemma.

Lemma 1 Let us now assume that

$$
\sup _{K} \mathbb{E}\left[\frac{N_{1}^{K}(0)}{K}\right]<+\infty, \quad \sup _{K} \mathbb{E}\left[\frac{N_{2}^{K}(0)}{K^{1+\gamma_{2}}}\right]<+\infty, \quad \sup _{K} \mathbb{E}\left[\frac{N_{3}^{K}(0)}{K^{1+\gamma_{2}+\gamma_{3}}}\right]<+\infty .
$$

then

$$
\sup _{K, t \in \mathbb{R}_{+}} \mathbb{E}\left[\frac{N_{1}^{K}(t)}{K}\right]<+\infty, \sup _{K, t \in \mathbb{R}_{+}} \mathbb{E}\left[\frac{N_{2}^{K}(t)}{K^{1+\gamma_{2}}}\right]<+\infty, \sup _{K, t \in \mathbb{R}_{+}} \mathbb{E}\left[\frac{N_{3}^{K}(t)}{K^{1+\gamma_{2}+\gamma_{3}}}\right]<+\infty .
$$

Proof The first assertion follows immediately.

From (8), we obtain that for all $t \geq 0$,

$$
\mathbb{E}\left[\frac{N_{2}^{K}(t)}{K^{1+\gamma_{2}}}\right]=\frac{\tau_{1}}{\tau_{2}} \mathbb{E}\left[\frac{N_{1}^{K}(0)}{K}\right]+\left(\mathbb{E}\left[\frac{N_{2}^{K}(0)}{K^{1+\gamma_{2}}}\right]-\frac{\tau_{1}}{\tau_{2}} \mathbb{E}\left[\frac{N_{1}^{K}(0)}{K}\right]\right) e^{-\tau_{2} K^{-\gamma_{2}} t}
$$

and the proof of the second assertion follows.

Similarly, straightforward computation yields

If $\tau_{3} K^{-\gamma_{3}} \neq \tau_{2} K^{-\gamma_{2}}$ then

$$
\begin{aligned}
\mathbb{E}\left[\frac{N_{3}^{K}(t)}{K^{1+\gamma_{2}+\gamma_{3}}}\right]= & \frac{2 p_{2}^{D} \tau_{1}}{\tau_{3}} \mathbb{E}\left[\frac{N_{1}^{K}(0)}{K}\right]-\beta_{K} e^{-\tau_{2} K^{-\gamma_{2}} t} \\
& +\left(\mathbb{E}\left[\frac{N_{3}^{K}(0)}{K^{1+\gamma_{2}+\gamma_{3}}}\right]-\frac{2 p_{2}^{D} \tau_{1}}{\tau_{3}} \mathbb{E}\left[\frac{N_{1}^{K}(0)}{K}\right]+\beta_{K}\right) e^{-\tau_{3} K^{-\gamma_{3}} t},
\end{aligned}
$$


with

$$
\beta_{K}=2 p_{2}^{D} \tau_{2} \frac{1}{\tau_{2} K^{\gamma_{3}-\gamma_{2}}-\tau_{3}}\left(\mathbb{E}\left[\frac{N_{2}^{K}(0)}{K^{1+\gamma_{2}}}\right]-\frac{\tau_{1}}{\tau_{2}} \mathbb{E}\left[\frac{N_{1}^{K}(0)}{K}\right]\right)<\infty
$$

If $\tau_{3} K^{-\gamma_{3}}=\tau_{2} K^{-\gamma_{2}}$ then

$$
\begin{aligned}
\mathbb{E}\left[\frac{N_{3}^{K}(t)}{\left.K^{1+\gamma_{2}+\gamma_{3}}\right]=}\right. & {\left[2 \tau_{2} p_{2}^{D} K^{-\gamma_{3}}\left(\mathbb{E}\left[\frac{N_{2}^{K}(0)}{K^{1+\gamma_{2}}}\right]-\frac{\tau_{1}}{\tau_{2}} \mathbb{E}\left[\frac{N_{1}^{K}(0)}{K}\right]\right) t\right.} \\
& \left.+\frac{\mathbb{E}\left[N_{3}^{K}(0)\right]}{K^{1+\gamma_{2}+\gamma_{3}}}-\frac{2 p_{2}^{D} \tau_{1}}{\tau_{2}} K^{\gamma_{2}-\gamma_{3}} \mathbb{E}\left[\frac{N_{1}^{K}(0)}{K}\right]\right] e^{-\tau_{2} K^{-\gamma_{2} t}} \\
& +\frac{2 p_{2}^{D} \tau_{1}}{\tau_{2}} K^{\gamma_{2}-\gamma_{3}} \mathbb{E}\left[\frac{N_{1}^{K}(0)}{K}\right],
\end{aligned}
$$

Hence the third assertion is proved.

3.2 Asymptotic behavior on a finite time interval

The parameter $K$ is defined as the order of magnitude of the martingale $N_{1}^{K}$ at time 0 .

Proposition 1 describes the dynamics of the process on a finite time interval. Its proof is left to the reader. It is standard and similar proofs will be given in the following section.

Proposition 1 Let us introduce the jump process $X^{K}$ defined for all $t \geq 0$ by

$$
X^{K}(t)=\left(\frac{N_{1}^{K}(t)}{K}, \frac{N_{2}^{K}(t)}{K^{1+\gamma_{2}}}, \frac{N_{3}^{K}(t)}{K^{1+\gamma_{2}+\gamma_{3}}}\right) .
$$

(i) Let us assume that there exists a vector $\left(x_{1}, 0,0\right) \in \mathbb{R}_{+}^{3}$ such that the sequence $\left(\frac{N_{1}^{K}(0)}{K}, \frac{N_{2}^{K}(0)}{K^{1+\gamma_{2}}}, \frac{N_{3}^{K}(0)}{K^{1+\gamma_{2}+\gamma_{3}}}\right)_{K \in \mathbb{N}^{*}}$ converges in law to $\left(x_{1}, 0,0\right)$ when $K$ tends to infinity and such that

$$
\sup _{K} \mathbb{E}\left[\frac{N_{1}^{K}(0)}{K}\right]<+\infty, \quad \sup _{K} \mathbb{E}\left[\frac{N_{2}^{K}(0)}{K^{1+\gamma_{2}}}\right]<+\infty \quad \text { and } \quad \sup _{K} \mathbb{E}\left[\frac{N_{3}^{K}(0)}{K^{1+\gamma_{2}+\gamma_{3}}}\right]<+\infty .
$$

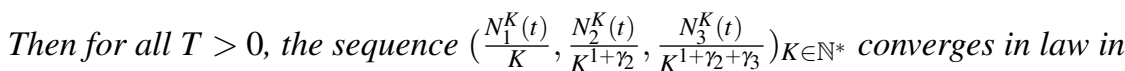
$\mathbb{D}\left([0, T], \mathbb{R}_{+}^{3}\right)$ to $\left(x_{1}, 0,0\right)$.

(ii) Let us assume that there exists a vector $\left(x_{1}, 0,0\right) \in \mathbb{R}_{+}^{3}$ such that the sequence $\left(\frac{N_{1}^{K}(0)}{K}, \frac{N_{2}^{K}(0)}{K}, \frac{N_{3}^{K}(0)}{K}\right)_{K \in \mathbb{N}^{*}}$ converges in law to $\left(x_{1}, 0,0\right)$ when $K$ tends to infinity and such that

$$
\sup _{K} \mathbb{E}\left[\frac{N_{1}^{K}(0)}{K}\right]<+\infty, \quad \sup _{K} \mathbb{E}\left[\frac{N_{2}^{K}(0)}{K}\right]<+\infty \quad \text { and } \quad \sup _{K} \mathbb{E}\left[\frac{N_{3}^{K}(0)}{K}\right]<+\infty .
$$

Then for all $T>0$, the sequence $\left(\frac{N_{1}^{K}(t)}{K}, \frac{N_{2}^{K}(t)}{K}, \frac{N_{3}^{K}(t)}{K}\right)_{K \in \mathbb{N}^{*}}$ converges in law in $\mathbb{D}\left([0, T], \mathbb{R}_{+}^{3}\right)$ to $x_{1}\left(1, \tau_{1} t, \frac{\tau_{2}}{2} t^{2}\right)$. 
Let us underline that at this time scale, assertion (i) shows that the two last components do not reach their equilibrium order, as observed in Figure 1. Assertion (ii) proves that the three cell types are only of order $K$ during the time interval $[0, T]$.

\section{Size-time multi-scale dynamics and asymptotic behavior}

A size renormalization of the stochastic process is not enough to understand the dynamics of the model. We need to change the time scale as observed in the simulations. Let us study the asymptotics for the process corresponding to the two time scales $K^{\gamma_{2}}$ and $K^{\gamma_{3}}$, when $K$ is large.

4.1 Asymptotic behavior at a time-scale of order $K^{\gamma_{2}}$

In this section, we study the system composed of the two first components at the time scale $K^{\gamma_{2}}$. To this end, let us introduce the jump process $Y^{K}$ defined for all $t \geq 0$ by

$$
Y^{K}(t)=\left(\frac{N_{1}^{K}\left(t K^{\gamma_{2}}\right)}{K}, \frac{N_{2}^{K}\left(t K^{\gamma_{2}}\right)}{K^{1+\gamma_{2}}}\right) .
$$

Let us note that at time $t=0$,

$$
\left(Y_{1}^{K}(0), Y_{2}^{K}(0)\right)=\left(X_{1}^{K}(0), X_{2}^{K}(0)\right) .
$$

The next theorem describes the approximating behavior of $Y^{K}$ when $K$ tends to infinity.

Theorem 1 Assume that there exists a vector $\left(x_{1}, x_{2}\right) \in \mathbb{R}_{+}^{2}$ such that the sequence $\left(Y^{K}(0)\right)_{K \in \mathbb{N}^{*}}$ converges in law to $\left(x_{1}, x_{2}\right)$ when $K$ tends to infinity and such that

$$
\sup _{K} \mathbb{E}\left[Y_{1}^{K}(0)^{2}+Y_{2}^{K}(0)^{2}\right]<\infty .
$$

Then for each $T>0$, the sequence $\left(Y^{K}\right)_{K \in \mathbb{N}^{*}}$ converges in law (and hence in probability) in $\mathbb{D}\left([0, T], \mathbb{R}_{+}^{2}\right)$ to the continuous function $y=\left(y_{1}, y_{2}\right)$ such that for all $t \geq 0$,

$$
\left\{\begin{array}{l}
y_{1}(t)=x_{1} \\
y_{2}(t)=\frac{\tau_{1} x_{1}}{\tau_{2}}+\left(x_{2}-\frac{\tau_{1} x_{1}}{\tau_{2}}\right) e^{-\tau_{2} t}
\end{array}\right.
$$

Proof By standard localization argument, use of Gronwall's Lemma and Doob's inequality, we easily prove (successively for the first and then for the second component) that for any $T>0$,

$$
\sup _{K} \mathbb{E}\left[\sup _{t \in[0, T]}\left(Y_{1}^{K}(t)^{2}+Y_{2}^{K}(t)^{2}\right)\right]<\infty .
$$

From (5) and (6), we can write

$$
\begin{aligned}
& Y_{1}^{K}(t)=Y_{1}^{K}(0)+\widehat{M}_{1}^{K}(t) \\
& Y_{2}^{K}(t)=Y_{2}^{K}(0)+\tau_{1} \int_{0}^{t} Y_{1}^{K}(u) d u-\tau_{2} \int_{0}^{t} Y_{2}^{K}(u) d u+\widehat{M}_{2}^{K}(t)
\end{aligned}
$$


where $\widehat{M}_{1}^{K}$ and $\widehat{M}_{2}^{K}$ are two square-integrable martingales satisfying

$$
\begin{aligned}
\left\langle\widehat{M}_{1}^{K}\right\rangle_{t} & =\frac{\tau_{1}}{K^{1-\gamma_{2}}} \int_{0}^{t} Y_{1}^{K}(u) d u, \\
\left\langle\widehat{M}_{2}^{K}\right\rangle_{t} & =\frac{2 \tau_{1}}{K^{1+\gamma_{2}}} \int_{0}^{t} Y_{1}^{K}(u) d u+\frac{\tau_{2}}{K} \int_{0}^{t} Y_{2}^{K}(u) d u, \\
\left\langle\widehat{M}_{1}^{K}, \widehat{M}_{2}^{K}\right\rangle_{t} & =-\frac{\tau_{1}}{K} \int_{0}^{t} Y_{1}^{K}(u) d u .
\end{aligned}
$$

It is standard to prove that the sequence of laws of $\left(Y^{K}\right)$ is tight (using the moment estimates (12)) and that the martingale parts go to 0 . The result follows using the method summarized for example in Bansaye and Meleard (2015). Each limiting value is proved to only charge the subset of continuous functions. Then introducing

$$
\phi_{t}(y)=\left(\begin{array}{c}
y_{1}(t)-y_{1}(0) \\
y_{2}(t)-y_{2}(0)-\int_{0}^{t}\left(\tau_{1} y_{1}(s)-\tau_{2} y_{2}(s)\right) d s
\end{array}\right)
$$

and using the uniform integrability of the sequence $\left(\phi_{t}\left(Y^{K}\right)\right)_{K}$, deduced from (12), we identify the limit as the unique continuous solution $y$ of the deterministic system defined by $y(0)=\left(x_{1}, x_{2}\right)$ and for all $t \geq 0, \phi_{t}(y)=0$.

That concludes the proof.

Remark 1 Since $\gamma_{2}<\gamma_{3}$, the time scale $K^{\gamma_{2}}$ is not large enough to observe the dynamics of the third component. The next proposition shows that at such a time scale, the third component converges to a trivial value.

Proposition 2 Under the same hypotheses as in Theorem 1, we assume furthermore that there exists $x_{3} \in \mathbb{R}_{+}$such that the sequence $\left(\frac{N_{3}^{K}(0)}{K^{1+\gamma_{2}+\gamma_{3}}}\right)_{K \in \mathbb{N}^{*}}$ converges in law to $x_{3}$ when $K$ tends to infinity and such that

$$
\sup _{K} \mathbb{E}\left[\left(\frac{N_{3}^{K}(0)}{K^{1+\gamma_{2}+\gamma_{3}}}\right)^{2}\right]<\infty .
$$

Then for each $T>0$, the sequence $\left(\frac{N_{3}^{K}\left(. K^{\gamma_{2}}\right)}{K^{1+\gamma_{2}+\gamma_{3}}}\right)_{K \in \mathbb{N}^{*}}$ converges in probability in $\mathbb{D}\left([0, T], \mathbb{R}_{+}\right)$ to $x_{3}$.

Proof Following (5) and (6), let us write the semimartingale decomposition of the process $Y_{3}^{K}=\frac{N_{3}^{K}\left(. K^{\gamma_{2}}\right)}{K^{1+\gamma_{2}+\gamma_{3}}}$. We have for any $t \leq T$,

$$
Y_{3}^{K}(t)=Y_{3}^{K}(0)+2 \tau_{2} p_{2}^{D} K^{\gamma_{2}-\gamma_{3}} \int_{0}^{t} Y_{2}^{K}(s) d s-\tau_{3} K^{\gamma_{2}-\gamma_{3}} \int_{0}^{t} Y_{3}^{K}(s) d s+\widehat{M}_{3}^{K}(t),
$$

where $\widehat{M}_{3}^{K}$ is a square-integrable martingale such that

$$
\left\langle\widehat{M}_{3}^{K}\right\rangle_{t}=2 \tau_{2} p_{2}^{D} K^{\gamma_{2}-\gamma_{3}} \int_{0}^{t} Y_{2}^{K}(s) d s+\tau_{3} K^{\gamma_{2}-\gamma_{3}} \int_{0}^{t} Y_{3}^{K}(s) d s .
$$

Let us recall that $\gamma_{2}<\gamma_{3}$, which makes $K^{\gamma_{2}-\gamma_{3}}$ tend to 0 when $K$ tends to infinity.

Using Theorem 1, we know that $Y_{2}^{K}$ converges to the continuous function $y_{2}$. By standard tightness argument, one can easily deduce that the process $Y_{3}^{K}$ converges in probability to $x_{3}$, on any finite time interval. 
4.2 Asymptotic behavior at a time-scale of order $K^{\gamma_{3}}$

In order to catch the long time dynamics of the third component we will study the process $N^{K}$ on the time scale $K^{\gamma_{3}}$. To this end, let us introduce the jump process $Z^{K}$ defined for all $t \geq 0$ by

$$
Z^{K}(t)=\left(\frac{N_{1}^{K}\left(t K^{\gamma_{3}}\right)}{K}, \frac{N_{2}^{K}\left(t K^{\gamma_{3}}\right)}{K^{1+\gamma_{2}}}, \frac{N_{3}^{K}\left(t K^{\gamma_{3}}\right)}{K^{1+\gamma_{2}+\gamma_{3}}}\right) .
$$

Note that we still have

$$
Z^{K}(0)=Y^{K}(0)=X^{K}(0)
$$

At this time scale, the second component has time to reach the equilibrium of its deterministic approximation by an average procedure. By an adaptation of the proof in Kang et al. (2014) to this specific framework, we are able to prove the following theorem.

Theorem 2 Assume that there exists $\left(x_{1}, x_{2}, x_{3}\right) \in \mathbb{R}_{+}^{3}$ such that the sequence $\left(Z^{K}(0)\right)_{K \in \mathbb{N}^{*}}$ converges in law to $\left(x_{1}, x_{2}, x_{3}\right)$ when $K$ tends to infinity and such that

$$
\sup _{K} \mathbb{E}\left[Z_{1}^{K}(0)\right]<+\infty, \quad \sup _{K} \mathbb{E}\left[Z_{2}^{K}(0)\right]<+\infty \quad \text { and } \quad \sup _{K} \mathbb{E}\left[Z_{3}^{K}(0)\right]<+\infty .
$$

Let $\Gamma_{2}^{K}$ be the $l_{m}\left(\mathbb{R}_{+}\right)$-valued random variable given by

$$
\Gamma_{2}^{K}([0, t] \times B)=\int_{0}^{t} \mathbf{1}_{\mathbf{B}}\left(\mathbf{Z}_{\mathbf{2}}^{\mathbf{K}}(\mathbf{s})\right) \mathbf{d s} .
$$

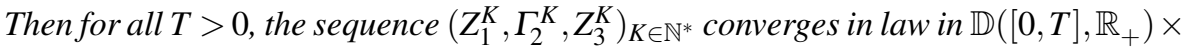
$l_{m}\left(\mathbb{R}_{+}\right) \times \mathbb{D}\left([0, T], \mathbb{R}_{+}\right)$to $\left(z_{1}, \delta_{z_{2}^{*}}\left(d z_{2}\right) d s, z_{3}\right)$. The functions $z_{1}$ and $z_{3}$ are defined for all $t \leq T$ by

$$
\left\{\begin{array}{l}
z_{1}(t)=x_{1} \\
z_{3}(t)=\frac{\tau_{2}}{\tau_{3}} z_{2}^{*}+\left(x_{3}-\frac{\tau_{2}}{\tau_{3}} z_{2}^{*}\right) e^{-\tau_{3} t}
\end{array}\right.
$$

and $z_{2}^{*}$ is the value of $y_{2}$ at infinity:

$$
z_{2}^{*}=\frac{\tau_{1} x_{1}}{\tau_{2}}
$$

Let us first state a lemma in which all moment estimates are gathered.

Lemma 2 Under Assumption (16), we obtain

$$
\sup _{K} \mathbb{E}\left[\sup _{t \in[0, T]} Z_{1}^{K}(t)\right]<+\infty ; \quad \forall t>0 \quad \sup _{K} \mathbb{E}\left[\int_{0}^{t} Z_{2}^{K}(s) d s\right]<+\infty
$$

and

$$
\sup _{K} \mathbb{E}\left[\sup _{t \in[0, T]} Z_{3}^{K}(t)\right]<+\infty
$$


Proof (Proof of Lemma 2) The first and third estimates are obtained by usual arguments (localization, Doob's inequality and Gronwall's Lemma). Let us focus on the second one.

By positivity and definition of the process $Z_{2}^{K}$, we have for any $t>0$

$$
Z_{2}^{K}(t)=Z_{2}^{K}(0)+\tau_{1} K^{\gamma_{3}-\gamma_{2}} \int_{0}^{t} Z_{1}^{K}(s) d s-\tau_{2} K^{\gamma_{3}-\gamma_{2}} \int_{0}^{t} Z_{2}^{K}(s) d s+\widetilde{M}_{2}^{K}(t)
$$

the latter term being a square-integrable martingale and

$$
\left\langle\widetilde{M}_{2}^{K}\right\rangle_{t}=\frac{1}{K^{1+\gamma_{2}}}\left(2 \tau_{1} K^{\gamma_{3}-\gamma_{2}} \int_{0}^{t} Z_{1}^{K}(s) d s+\tau_{2} K^{\gamma_{3}} \int_{0}^{t} Z_{2}^{K}(s) d s\right) .
$$

In particular,

$$
\mathbb{E}\left[\int_{0}^{t} Z_{2}^{K}(s) d s\right]=\frac{1}{\tau_{2}} K^{\gamma_{2}-\gamma_{3}}\left(\mathbb{E}\left[Z_{2}^{K}(0)\right]-\mathbb{E}\left[Z_{2}^{K}(t)\right]\right)+\frac{\tau_{1}}{\tau_{2}} \int_{0}^{t} \mathbb{E}\left[Z_{1}^{K}(s)\right] d s .
$$

Assumptions (16) and Lemma 1 ensure that the first term goes to 0 as $K$ tends to infinity and the third term is bounded uniformly in $K$. That allows to conclude.

Proof (Proof of Theorem 2) Let $\Gamma^{K}$ be the occupation measure of $Z^{K}$, a random measure belonging to the space $l_{m}\left(\mathbb{R}_{+}\right)$of positive measures on $[0, \infty) \times \mathbb{R}_{+}$with mass $t$ on $[0, t] \times \mathbb{R}_{+}$, and defined for all Borelian set $B$ and for $t>0$ by

$$
\Gamma^{K}([0, t] \times B)=\int_{0}^{t} \mathbf{1}_{\mathbf{B}}\left(\mathbf{Z}^{\mathbf{K}}(\mathbf{s})\right) \mathbf{d s} .
$$

Using Lemma 3 (cf. Appendix), we obtain that $\left(\Gamma^{K}\right)_{K}$ is relatively compact in $l_{m}\left(\mathbb{R}_{+}\right)$ endowed with a weak topology generated by the class of test functions defined in (43).

Let us denote by $\Gamma \in l_{m}\left(\mathbb{R}_{+}^{3}\right)$ a limiting value of $\left(\Gamma^{K}\right)_{K}$. Using Lemma 1.4 in Kurtz (1992), one can show that there exists a $\mathscr{P}\left(\mathbb{R}_{+}^{3}\right)$-valued process $\gamma_{s}$ such that

$$
\Gamma(d u \times d s)=\gamma_{s}(d u) d s .
$$

Let us now introduce the function $F^{K}$, for all $u \in \mathbb{R}_{+}^{3}$,

$$
F^{K}(u)=\left(1+\frac{1}{K^{\gamma_{2}}}\right) \tau_{2} u_{2}-\tau_{3} u_{3}
$$

Then for all $t \geq 0$,

$$
\begin{aligned}
Z_{3}^{K}(t) & =Z_{3}^{K}(0)+\left(1+\frac{1}{K^{\gamma_{2}}}\right) \tau_{2} \int_{0}^{t} Z_{2}^{K}(s) d s-\tau_{3} \int_{0}^{t} Z_{3}^{K}(s) d s+\widetilde{M}_{3}^{K}(t) \\
& =Z_{3}^{K}(0)+\int_{0}^{t} F^{K}\left(Z^{K}(s)\right) d s+\widetilde{M}_{3}^{K}(t) \\
Z_{1}^{K}(t) & =Z_{1}^{K}(0)+\widetilde{M}_{1}^{K}(t)
\end{aligned}
$$


with $\left(\widetilde{M}_{1}^{K}, \widetilde{M}_{3}^{K}\right)$ independent martingales such that for all $t \geq 0$,

$$
\begin{aligned}
& <\widetilde{M}_{1}^{K}>_{t}=K^{\gamma_{3}-1} \int_{0}^{t} 2 \tau_{1} Z_{1}^{K}(s) d s \\
& <\widetilde{M}_{3}^{K}>_{t}=K^{-\left(1+\gamma_{2}+\gamma_{3}\right)} \int_{0}^{t}\left(2\left(1+\frac{1}{K^{\gamma_{2}}}\right) \tau_{2} Z_{2}^{K}(s)+\tau_{3} Z_{3}^{K}(s)\right) d s .
\end{aligned}
$$

By usual arguments involving Lemma 2, one can prove that the sequences of processes $\left(Z_{3}^{K}\right)_{K}$ and $\left(Z_{1}^{K}\right)_{K}$ are uniformly tight in $\mathbb{D}\left([0, T], \mathbb{R}_{+}\right)$. Let us also note that the distributions of any limiting value only charge processes with a.s. continuous trajectories.

Furthermore by Doob's inequality

$$
\mathbb{E}\left[\sup _{t \leq T}\left|\widetilde{M}_{3}^{K}(t)\right|^{2}\right] \leq 4 \mathbb{E}\left[<\widetilde{M}_{3}^{K}>_{T}\right] .
$$

Using (23) and Lemma 2, we obtain that $\lim _{K \rightarrow \infty} \mathbb{E}\left[\sup _{t \in[0, T]}\left|\widetilde{M}_{3}^{K}(t)\right|^{2}\right]=0$ and a similar property for $\widetilde{M}_{1}^{K}$ since $\gamma_{3}<1$. Then, we deduce from Markov's inequality, that the processes $\left(\widetilde{M}_{3}^{K}\right)_{K}$ and $\left(\widetilde{M}_{1}^{K}\right)_{K}$ converge in probability for the uniform norm to 0 . Hence they converge in law in $\mathbb{D}\left([0, T], \mathbb{R}_{+}\right)$to 0 .

Adding all these asymptotic behaviors, we deduce that there exists a subsequence of $\left(Z_{1}^{K}, Z_{3}^{K}\right)$ converging in law in $\mathbb{D}\left([0, T], \mathbb{R}_{+}^{2}\right)$ to the deterministic limit $\left(z_{1}, z_{3}\right)$ defined for all $t \geq 0$ by

$$
\begin{aligned}
& z_{1}(t)=x_{1} \\
& z_{3}(t)=x_{3}+\int_{\mathbb{R}_{+}^{3} \times[0, t]}\left(\tau_{2} u_{2}-\tau_{3} u_{3}\right) \gamma_{s}(d u) d s .
\end{aligned}
$$

Then by convergence of $\left(Z_{1}^{K}\right)_{K},\left(Z_{3}^{K}\right)_{K}$ and $\Gamma^{K}$, one can easily deduce that

$$
\gamma_{s}(d u)=\delta_{x_{1}}\left(d u_{1}\right) \delta_{z_{3}(s)}\left(d u_{3}\right) \tilde{\gamma}_{s}\left(u_{1}, u_{3}, d u_{2}\right) .
$$

We have now to identify the measures $\tilde{\gamma}_{s}, s \in[0, T]$.

Let us write the generator of the process $Z^{K}$ applied on the second component. For $h \in C_{c}^{\infty}$, it is given for $u \in \mathbb{R}_{+}^{3}$ by

$$
\begin{aligned}
\mathscr{L}_{2}^{K}(h)(u)= & \left(h\left(u_{2}+2 K^{-\left(1+\gamma_{2}\right)}\right)-h\left(u_{2}\right)\right) \frac{\tau_{1}}{2} u_{1} K^{1+\gamma_{3}} \\
& +\left(h\left(u_{2}+K^{-\left(1+\gamma_{2}\right)}\right)-h\left(u_{2}\right)\right) p_{2}^{R} \tau_{2} u_{2} K^{1+\gamma_{2}+\gamma_{3}} \\
& +\left(h\left(u_{2}-K^{-\left(1+\gamma_{2}\right)}\right)-h\left(u_{2}\right)\right) p_{2}^{D} \tau_{2} u_{2} K^{1+\gamma_{2}+\gamma_{3}} .
\end{aligned}
$$

Introducing the function $g$ defined for $u \in \mathbb{R}_{+}^{3}$ by $g(u)=u_{1} \tau_{1}-\tau_{2} u_{2}$, we obtain, by a Taylor expansion, that for all $h \in C_{c}^{\infty}$

$$
\lim _{K \rightarrow \infty} \sup _{u \in \mathbb{R}_{+}^{3}}\left|K^{\gamma_{2}-\gamma_{3}} \mathscr{L}_{2}^{K}(h)(u)-g(u) h^{\prime}\left(u_{2}\right)\right|=0 .
$$


Using (19), Lemma 3 and the same arguments as above, we obtain that the sequence of processes $\left(K^{\gamma_{2}-\gamma_{3}}\left(h\left(Z_{2}^{K}(t)\right)-h\left(Z_{2}^{K}(0)\right)-\int_{0}^{t} \mathscr{L}_{2}^{K}(h)\left(Z^{K}(s)\right) d s\right), \quad t \in\right.$ $[0, T])$ converges in law in $\mathbb{D}([0, T], \mathbb{R})$ to 0 . On the other hand, using that $h$ is bounded and (24) and $g h^{\prime} \in C_{b}$ (since $h$ has compact support), we easily see that this sequence also converges to

$$
-\int_{0}^{t}\left(\tau_{1} x_{1}-\tau_{2} u_{2}\right) h^{\prime}\left(u_{2}\right) \gamma_{s}\left(x_{1}, z_{3}(s), d u_{2}\right) d s
$$

We deduce that for any $t \in[0, T]$, for any $h \in C_{c}^{\infty}$

$$
\int_{0}^{t}\left(\tau_{1} x_{1}-\tau_{2} u_{2}\right) h^{\prime}\left(u_{2}\right) \tilde{\gamma}_{s}\left(x_{1}, z_{3}(s), d u_{2}\right) d s=0
$$

The only probability measure which can realize such equality for all $h \in C_{c}^{\infty}$ is the Dirac measure such that for all $u_{2},\left(\tau_{1} x_{1}-\tau_{2} u_{2}\right)=0$. It implies that

$$
\tilde{\gamma}_{s}\left(x_{1}, z_{3}(s), d u_{2}\right)=\delta_{\frac{\tau_{1} x_{1}}{\tau_{2}}}\left(d u_{2}\right) .
$$

To end the proof, we solve the equation satisfied by $z_{3}(t)$ and obtain that, for all $t \geq 0$,

$$
z_{3}(t)=\frac{\tau_{1} x_{1}}{\tau_{3}}+\left(x_{3}-\frac{\tau_{1} x_{1}}{\tau_{3}}\right) e^{-\tau_{3} t}
$$

Hence we have uniquely identified the limit of any converging subsequence. That ends the proof.

\section{Amplified fluctuations}

In this section, we will quantify the large fluctuations observed on the simulations. As pointed out above, each component has its own typical size and time scale. Hence, we will study separately the fluctuations of the second and third types. Classical results easily imply that the first component, on time scale of order 1 , behaves as a Brownian motion: for large $K$, for all $t \in[0, T]$,

$$
N_{1}^{K}(t) \sim x_{1} K+\sqrt{K} \sqrt{\tau_{1} x_{1}} B_{t}
$$

The originality of our results concerns the large fluctuations of the two last types due to the amplification of these type 1 cell size fluctuations. 
5.1 The large fluctuations of the second type

As seen in Subsection 4.1, the typical size scale (respectively time scale) of the second type is $K^{1+\gamma_{2}}$ (respectively $K^{\gamma_{2}}$ ) and the first order asymptotics relative to this time scale is given by the function $y$ defined by (11). We are also able to give an expansion of the process at the second and third orders on such time scale.

Theorem 3 Let define the sequence $\left(U^{K}\right)_{K}$ by for all $t \geq 0$,

$$
U^{K}(t)=K^{\left(1-\gamma_{2}\right) / 2}\left(Y^{K}(t)-y(t)\right) .
$$

(i) Assume that there exists $U_{0}=\left(U_{0}^{(1)}, U_{0}^{(2)}\right) \in \mathbb{R}^{2}$ such that $\left(U^{K}(0)\right)_{K \in \mathbb{N}^{*}}$ converges in law to $U_{0}$ and that

$$
\sup _{K} \mathbb{E}\left[U_{1}^{K}(0)^{2}+U_{2}^{K}(0)^{2}\right]<+\infty .
$$

Then for each $T>0$, the sequence $\left(U^{K}\right)_{K \in \mathbb{N}^{*}}$ converges in law in $\mathbb{D}\left([0, T], \mathbb{R}^{2}\right)$ to the process $U=\left(U_{1}, U_{2}\right)$ defined for all $t \geq 0$ by

$$
\begin{aligned}
& U_{1}(t)=U_{0}^{(1)}+\sqrt{\tau_{1} x_{1}} B_{1}(t), \\
& U_{2}(t)=U_{0}^{(2)}+\tau_{1} \int_{0}^{t} U_{1}(s) d s-\tau_{2} \int_{0}^{t} U_{2}(s) d s,
\end{aligned}
$$

where $B_{1}$ is a standard Brownian motion.

(ii) Furthermore, the sequence $\left(W_{2}^{K}\right)_{K \in \mathbb{N}^{*}}$ defined by for all $t \in[0, T]$,

$$
W_{2}^{K}(t)=K^{\gamma_{2} / 2}\left[U_{2}^{K}(t)-U_{0}^{(2)}-\tau_{1} \int_{0}^{t} U_{1}^{K}(s) d s+\tau_{2} \int_{0}^{t} U_{2}^{K}(s) d s\right]
$$

converges in law in $\mathbb{D}([0, T], \mathbb{R})$, for each $T>0$, to the process $\left(\sqrt{\tau_{2} y_{2}(t)} B_{2}(t), \quad t \in\right.$ $[0, T])$ where $B_{2}$ is a standard Brownian motion independent of the process $B_{1}$.

From this theorem, we can deduce the following expansion, which quantifies the large waves of fluctuations. Assuming that $U_{0}$ is equal to zero, we obtain for all $t$ and large $K$,

$N_{2}^{K}(t) \sim K^{1+\gamma_{2}} y_{2}\left(t K^{-\gamma_{2}}\right)+K^{\left(1+3 \gamma_{2}\right) / 2} U_{2}\left(t K^{-\gamma_{2}}\right)+K^{\left(1+2 \gamma_{2}\right) / 2} \sqrt{\tau_{2} y_{2}\left(t K^{-\gamma_{2}}\right)} B_{2}\left(t K^{-\gamma_{2}}\right)$

where for all $t$

$$
U_{2}(t)=\tau_{1} \sqrt{\tau_{1} x_{1}} \int_{0}^{t} B_{1}(s) d s-\tau_{2} \int_{0}^{t} U_{2}(s) d s
$$

and $B_{1}, B_{2}$ are independent Brownian motions. 
Proof (of Theorem 3.) (i) First we deduce from (25) with similar arguments as above that

$$
\sup _{K} \mathbb{E}\left[\sup _{t \in[0, T]}\left(U_{1}^{K}(t)^{2}+U_{2}^{K}(t)^{2}\right)\right]<+\infty .
$$

The tightness of the families $\left(\sup _{t \leq T}\left|U_{1}^{K}(t)\right|\right)_{K}$ and $\left(\sup _{t \leq T}\left|U_{2}^{K}(t)\right|\right)_{K}$ immediately follows.

We consider the semi-martingale decomposition of $\left(U^{K}\right)$ and write

$$
U_{i}^{K}(t)=U_{i}^{K}(0)+A_{i}^{K}(t)+K^{\frac{1-\gamma_{2}}{2}} \widehat{M}_{i}^{K}(t)
$$

where $\widehat{M}^{K}$ has been defined in (13), $A_{1}^{K}=0$ and $A_{2}^{K}(t)=\tau_{1} \int_{0}^{t} U_{1}^{K}(s) d s-\tau_{2} \int_{0}^{t} U_{2}^{K}(s) d s$.

Thanks to the above moment estimates, it is almost immediate to prove that the finite variation processes $<K^{\frac{1-\gamma_{2}}{2}} \widehat{M}_{i}^{K}>$ and $A_{2}^{K}$ satisfy the Aldous condition. Thanks to Aldous and Rebolledo criteria (see Joffe and Metivier (1986) and Bansaye and Meleard (2015)), the uniform tightness of $\mathscr{L}\left(U^{K}\right)$ in $\mathscr{P}\left(\mathbb{D}\left([0, T], \mathbb{R}^{2}\right)\right)$ follows.

We denote by simplicity by the same notation $\left(U^{K}\right)_{K}$ a subsequence converging in law in $\mathbb{D}\left([0, T], \mathbb{R}^{2}\right)$. Let $Q$ be the limiting value of $\left(\mathscr{L}\left(U^{K}\right)\right)_{K}$. It is easy to observe that

$$
\sup _{t \in[0, T]}\left\|\Delta U^{K}(t)\right\| \leq 2 K^{-\left(1+\gamma_{2}\right) / 2}
$$

with $\Delta x(t)=x(t)-x\left(t^{-}\right)$.

Therefore, by continuity of the mapping $x \rightarrow \sup _{t \in[0, T]}\|\Delta x(t)\|$ from $\mathbb{D}\left([0, T], \mathbb{R}^{2}\right)$ into $\mathbb{R}_{+}$, the probability measure $Q$ only charges the processes with continuous paths.

The extended generator of $U^{K}$ is defined for functions $f \in C_{b}^{2}\left(\mathbb{R}^{2}, \mathbb{R}\right)$ as: $\forall u \in \mathbb{R}^{2}$,

$$
\begin{aligned}
\mathscr{L}^{K}(f, t)(u) & =\left(f\left(u_{1}+K^{-\left(1+\gamma_{2}\right) / 2}, u_{2}\right)-f(u)\right) \frac{\tau_{1}}{2} K^{1+\gamma_{2}}\left(K^{-\left(1-\gamma_{2}\right) / 2} u_{1}+x_{1}\right) \\
+ & \left(f\left(u_{1}-K^{-\left(1+\gamma_{2}\right) / 2}, u_{2}+2 K^{-\left(1+3 \gamma_{2}\right) / 2}\right)-f(u)\right) \frac{\tau_{1}}{2} K^{1+\gamma_{2}}\left(K^{-\left(1-\gamma_{2}\right) / 2} u_{1}+x_{1}\right) \\
+ & \left(f\left(u_{1}, u_{2}+K^{-\left(1+3 \gamma_{2}\right) / 2}\right)-f(u)\right) p_{2}^{R} \tau_{2} K^{1+2 \gamma_{2}}\left(K^{-\left(1-\gamma_{2}\right) / 2} u_{2}+y_{2}(t)\right) \\
& +\left(f\left(u_{1}, u_{2}-K^{-\left(1+3 \gamma_{2}\right) / 2}\right)-f(u)\right) p_{2}^{D} \tau_{2} K^{1+2 \gamma_{2}}\left(K^{-\left(1-\gamma_{2}\right) / 2} u_{2}+y_{2}(t)\right) \\
& -K^{\left(1-\gamma_{2}\right) / 2} \partial_{2} f(u)\left(\tau_{1} x_{1}-\tau_{2} y_{2}(t)\right) .
\end{aligned}
$$

By a Taylor's expansion, we easily obtain that $\forall f \in C_{b}^{2}\left(\mathbb{R}^{2}, \mathbb{R}\right)$,

$$
\lim _{K \rightarrow \infty} \sup _{(u, t) \in \mathbb{R}^{2} \times \mathbb{R}_{+}}\left|\mathscr{L}^{K}(f, t)(u)-\left(\frac{\tau_{1}}{2} x_{1} \partial_{1}^{2} f(u)+\left(\tau_{1} u_{1}-\tau_{2} u_{2}\right) \partial_{2} f(u)\right)\right|=0 .
$$


On the other hand, let us define, for $f \in C_{b}^{2}\left(\mathbb{R}^{2}, \mathbb{R}\right), u \in \mathbb{D}\left([0, T], \mathbb{R}^{2}\right)$ and $t \in[0, T]$, the function $\xi_{t}^{K, f}$ by

$$
\xi_{t}^{K, f}(u)=f\left(u_{t}\right)-f\left(u_{0}\right)-\int_{0}^{t} \mathscr{L}^{K}(f, s)\left(u_{s}\right) d s .
$$

Then, by (28), Dynkin's formula and (27), we can easily prove that the processes $\left(\xi_{t}^{K, f}\left(U^{K}\right)\right)_{K}$ are uniformly integrable martingales.

Therefore by standard arguments (cf. Ethier and Kurtz (1986) p.225, Bansaye and Meleard (2015) p.25), the limiting process under $Q$ is continuous and satisfies the following martingale problem: for all $f \in C_{b}^{2}\left(\mathbb{R}^{2}, \mathbb{R}\right)$,

$$
f(U(t))-f\left(U_{0}\right)-\int_{0}^{t}\left(\frac{\tau_{1}}{2} x_{1} \partial_{1}^{2} f(U(s))+\left(\tau_{1} U_{1}(s)-\tau_{2} U_{2}(s)\right) \partial_{2} f(U(s))\right) d s
$$

is a martingale.

We conclude using a representation theorem (cf. Ikeda and Watanabe (1989) p.84) that for each $T>0$, the sequence $\left(U^{K}\right)_{K \in \mathbb{N}^{*}}$ converges in law in $\mathbb{D}\left([0, T], \mathbb{R}^{2}\right)$ to the process $U=\left(U_{1}, U_{2}\right)$, unique solution of the following stochastic differential system: for all $t \in[0 ; T]$,

$$
\begin{aligned}
& U_{1}(t)=U_{0}^{(1)}+\sqrt{\tau_{1} x_{1}} B_{1}(t), \\
& U_{2}(t)=U_{0}^{(2)}+\tau_{1} \int_{0}^{t} U_{1}(s) d s-\tau_{2} \int_{0}^{t} U_{2}(s) d s,
\end{aligned}
$$

with $B_{1}$ a Brownian motion.

(ii) Let us now expand the second component to the next order. We deduce from (13) that

$$
\begin{aligned}
U_{1}^{K}(.)-U^{K}(0) & =K^{\left(1-\gamma_{2}\right) / 2} \widehat{M}_{1}^{K}(.), \\
W_{2}^{K}(.) & =\sqrt{K} \widehat{M}_{2}^{K}(.) .
\end{aligned}
$$

Using (14), (12) and applying Theorem 7.1.4 of Ethier and Kurtz (1986), we conclude the proof.

5.2 The large fluctuations of the third type

Let us now study the fluctuation process associated with the largest fluctuation scale of the third component. We have seen in Theorem 2 that at the time scale $K^{\gamma_{3}}$, the size of the population process of the third type is of order of magnitude $K^{1+\gamma_{2}+\gamma_{3}}$. In the usual setting, the Central Limit Theorem would lead to fluctuations of order $K^{\left(1+\gamma_{2}+\gamma_{3}\right) / 2}$. We will see in the next theorem that they are of order $K^{\left(1+2 \gamma_{2}+3 \gamma_{3}\right) / 2} \gg$ $K^{\left(1+\gamma_{2}+\gamma_{3}\right) / 2}$. 
Using (17), (18) and (20), we know that for all $t \geq 0$,

$$
\begin{aligned}
\left(Z_{3}^{K}(t)-z_{3}(t)\right)= & \left(Z_{3}^{K}(0)-z_{3}(0)\right)+\tau_{2} \int_{0}^{t}\left(Z_{2}^{K}(s)-z_{2}^{*}\right) d s-\tau_{3} \int_{0}^{t}\left(Z_{3}^{K}(s)-z_{3}(s)\right) d s \\
& +\tau_{2} \frac{1}{K^{\gamma / 2}} \int_{0}^{t}\left(Z_{2}^{K}(s)-z_{2}^{*}\right) d s+\widetilde{M}_{3}^{K}(t)
\end{aligned}
$$

where

$$
\tau_{2} \int_{0}^{t}\left(Z_{2}^{K}(s)-z_{2}^{*}\right) d s=\tau_{1} \int_{0}^{t}\left(Z_{1}^{K}(s)-x_{1}\right) d s-\frac{Z_{2}^{K}(t)-Z_{2}^{K}(0)}{K^{\gamma_{3}-\gamma_{2}}}+\frac{\widetilde{M}_{2}^{K}(t)}{K^{\gamma_{3}-\gamma_{2}}}
$$

Our goal is to quantify the effect of the first component fluctuations on the dynamics of the third component. Considering the expressions of the martingale quadratic variation (22) imposes the choice of the rescaling parameter $K^{\left(1-\gamma_{3}\right) / 2}$ in front of $\left(Z_{1}^{K}(t)-x_{1}\right)$. We will see that to keep the effect of the first component on the third component, we need to rescale $\left(Z_{2}^{K}(t)-Z_{2}^{K}(0)\right)$ by $\frac{K^{\left(1-\gamma_{3}\right) / 2}}{K^{\gamma_{3}-\gamma_{2}}}$ and $\left(Z_{3}^{K}(t)-z_{3}(t)\right)$ by $K^{\left(1-\gamma_{3}\right) / 2}$.

Let us now state the main theorem of this section.

Theorem 4 Let us define the three processes, for all $t \geq 0$,

$$
\left\{\begin{array}{l}
V_{1}^{K}(t)=K^{\left(1-\gamma_{3}\right) / 2}\left(Z_{1}^{K}(t)-x_{1}\right) \\
V_{2}^{K}(t)=\frac{K^{\left(1-\gamma_{3}\right) / 2}}{K^{\gamma_{3}-\gamma_{2}}}\left(Z_{2}^{K}(t)-Z_{2}^{K}(0)\right) \\
V_{3}^{K}(t)=K^{\left(1-\gamma_{3}\right) / 2}\left(Z_{3}^{K}(t)-z_{3}(t)\right)
\end{array}\right.
$$

We assume that

$$
\sup _{K} \mathbb{E}\left[V_{1}^{K}(0)^{4}\right]<+\infty \quad ; \quad \sup _{K} \mathbb{E}\left[Z_{2}^{K}(0)^{2}\right]<+\infty .
$$

(i) Then for all $T \geq 0$,

$$
\lim _{K \rightarrow \infty} \mathbb{E}\left[\sup _{t \in[0, T]} V_{2}^{K}(t)^{2}\right]=0
$$

(ii) We assume moreover that there exists $V_{0}=\left(V_{0}^{(1)}, V_{0}^{(3)}\right)$ a $\mathbb{R}^{2}$-valued random vector such that the sequence $\left(V_{1}^{K}(0), V_{3}^{K}(0)\right)_{K \in \mathbb{N}^{*}}$ converges in law to $V_{0}$ and such that

$$
\sup _{K} \mathbb{E}\left[\left|V_{3}^{K}(0)\right|\right]<+\infty .
$$

Then for all $T>0$, the sequence $\left(V_{1}^{K}, V_{3}^{K}\right)_{K \in \mathbb{N}^{*}}$ converges in law in $\mathbb{D}\left([0, T], \mathbb{R}^{2}\right)$ to $\left(V_{1}, V_{3}\right)$ such that for all $t$,

$$
V_{1}(t)=V_{0}^{(1)}+\sqrt{\tau_{1} x_{1}} W_{1}(t)
$$




$$
V_{3}(t)=V_{0}^{(3)}+\tau_{1} \int_{0}^{t} V_{1}(s) d s-\tau_{3} \int_{0}^{t} V_{3}(s) d s,
$$

where $W_{1}$ is a standard Brownian motion.

Let us interpret the result $(i i)$ in terms of fluctuations. Assuming that the initial vector $V_{0}$ is equal to zero, we obtain that for any $t$ and large $K$,

$$
N_{3}^{K}(t) \sim K^{1+\gamma_{2}+\gamma_{3}} z_{3}\left(t K^{-\gamma_{3}}\right)+K^{\left(1+2 \gamma_{2}+3 \gamma_{3}\right) / 2} V_{3}\left(t K^{-\gamma_{3}}\right)
$$

where for all $t$,

$$
V_{3}(t)=\tau_{1} \sqrt{\tau_{1} x_{1}} \int_{0}^{t} W_{1}(s) d s-\tau_{3} \int_{0}^{t} V_{3}(s) d s
$$

and $W_{1}$ is a standard Brownian motion.

The order of magnitude appearing in the fluctuation second order term (34) summarizes the cumulative effects of the third dynamics driven by the fluctuations of the first level. That can explain the exceptionally large fluctuations observed for the size of terminal cells populations, in hematopoietic systems.

As a first step, we will prove that the sequence of processes $\left(V_{2}^{K}\right)_{K}$ converges to 0 uniformly in $\mathbb{L}^{2}$ on any finite time interval.

Proof (i) Using (18), we obtain that, for all $t \geq 0$,

$$
\begin{aligned}
V_{2}^{K}(t) & =K^{\frac{1-3 \gamma_{3}}{2}+\gamma_{2}}\left(Z_{2}^{K}(t)-Z_{2}^{K}(0)\right) \\
& =\tau_{1} \int_{0}^{t} V_{1}^{K}(s) d s-\tau_{2} K^{\gamma_{3}-\gamma_{2}} \int_{0}^{t} V_{2}^{K}(s) d s+\mathscr{R}^{K}(t),
\end{aligned}
$$

where $\mathscr{R}^{K}$ is the square-integrable martingale defined by, for all $t \geq 0$,

$$
\mathscr{R}_{t}^{K}=K^{\frac{1-3 \gamma_{3}}{2}+\gamma_{2}} \widetilde{M}_{2}^{K}(t)
$$

and satisfying for all $t$,

$$
\left\langle\mathscr{R}^{K}\right\rangle_{t}=K^{-2 \gamma_{3}}\left(\tau_{1} \int_{0}^{t} Z_{1}^{K}(s) d s+\tau_{2} K^{\gamma_{2}} \int_{0}^{t} Z_{2}^{K}(s) d s\right) .
$$

Let us first show that for all $t>0$,

$$
\sup _{K} \mathbb{E}\left[\int_{0}^{t} V_{2}^{K}(s)^{4} d s\right]<\infty .
$$

Itô's formula immediately implies that $\forall t \geq 0$,

$$
\begin{aligned}
V_{2}^{K}(t)^{4}= & V_{2}^{K}(0)^{4}+4 \int_{0}^{t} V_{2}^{K}(s)^{3} d \mathscr{R}_{s}^{K}+4 \int_{0}^{t} V_{2}^{K}(s)^{3}\left(\tau_{1} V_{1}^{K}(s)-K^{\gamma_{3}-\gamma_{2}} \tau_{2} V_{2}^{K}(s)\right) d s \\
& +6 \int_{0}^{t} V_{2}^{K}(s)^{2} d\left\langle\mathscr{R}^{K}\right\rangle_{s} .
\end{aligned}
$$


By standard localization arguments, we prove using (31) that for any $t \geq 0$,

$$
\sup _{K} \mathbb{E}\left[\int_{0}^{t} V_{1}^{K}(s)^{4} d s\right]<\infty
$$

Let us now introduce the stopping time

$$
T_{n}=\inf \left\{t \geq 0,\left|V_{2}^{K}(t)\right| \geq n\right\} .
$$

Then, applying the following inequality

$$
\begin{aligned}
4\left(\tau_{1} v_{1} v_{2}-K^{\gamma_{3}-\gamma_{2}} \tau_{2} v_{2}^{2}\right) & =4 \tau_{2} K^{\gamma_{3}-\gamma_{2}}\left(\left[\frac{v_{1} \tau_{1}}{2 \tau_{2} K^{\gamma_{3}-\gamma_{2}}}\right]^{2}-\left[v_{2}-\frac{v_{1} \tau_{1}}{2 \tau_{2} K^{\gamma_{3}-\gamma_{2}}}\right]^{2}\right) \\
& \leq \frac{v_{1}^{2} \tau_{1}^{2}}{\tau_{2} K^{\gamma_{3}-\gamma_{2}}}
\end{aligned}
$$

to $v_{1}=V_{1}^{K}(s)$ and $v_{2}=V_{2}^{K}(s)$, we obtain the following upper-bound

$$
\begin{aligned}
\mathbb{E}\left[V_{2}^{K}\left(t \wedge T_{n}\right)^{4}\right] \leq \mathbb{E}\left[V_{2}^{K}(0)^{4}\right] & +\frac{\tau_{1}^{2}}{\tau_{2} K^{\gamma_{3}-\gamma_{2}}} \int_{0}^{t \wedge T_{n}} \mathbb{E}\left[V_{2}^{K}(s)^{2} V_{1}^{K}(s)^{2}\right] d s \\
& +6 \mathbb{E}\left[\int_{0}^{t \wedge T_{n}} V_{2}^{K}(s)^{2} d\left\langle\mathscr{R}^{K}\right\rangle_{s}\right]
\end{aligned}
$$

Using (36), we obtain for all $t \in[0, T]$,

$\mathbb{E}\left[\int_{0}^{t \wedge T_{n}} V_{2}^{K}(s)^{2} d\left\langle\mathscr{R}^{K}\right\rangle_{s}\right]=K^{\gamma_{2}-2 \gamma_{3}} \mathbb{E}\left[\int_{0}^{t \wedge T_{n}} V_{2}^{K}(s)^{2}\left(K^{-\gamma_{2}} \tau_{1} Z_{1}^{K}(s)+\tau_{2} Z_{2}^{K}(s)\right) d s\right]$.

Writing $Z_{2}^{K}$ in function of $V_{2}^{K}$, we find the following upper bound,

$$
\begin{aligned}
\mathbb{E}\left[\int_{0}^{t \wedge T_{n}} V_{2}^{K}(s)^{2} d\left\langle\mathscr{R}^{K}\right\rangle_{s}\right] & \leq\left(\tau_{1} K^{-2 \gamma_{3}}+\tau_{2} K^{-\left(1+\gamma_{3}\right) / 2}+\tau_{2} K^{\gamma_{2}-2 \gamma_{3}}\right) \int_{0}^{t \wedge T_{n}} \mathbb{E}\left[V_{2}^{K}(s)^{4}\right] d s \\
& +\tau_{1} K^{-2 \gamma_{3}} \int_{0}^{t \wedge T_{n}} \mathbb{E}\left[Z_{1}^{K}(s)^{2}\right] d s \\
& +\tau_{2}\left(K^{-\left(1+\gamma_{3}\right) / 2}+K^{\gamma_{2}-2 \gamma_{3}}\right)\left(\mathbb{E}\left[Z_{2}^{K}(0)^{2}\right]+1\right) T
\end{aligned}
$$

We deduce from (39) using Lemma 2, (31) and Gronwall's Lemma that $\forall t \in$ $[0, T]$

$$
\sup _{K} \mathbb{E}\left[\int_{0}^{t \wedge T_{n}} V_{2}^{K}(s)^{4} d s\right]<\infty
$$

Let us now come back to the proof of (32). Itô's formula yields

$$
V_{2}^{K}(t)^{2}=2 \int_{0}^{t} V_{2}^{K}(s) d \mathscr{R}_{s}^{K}+2 \int_{0}^{t} V_{2}^{K}(s)\left(\tau_{1} V_{1}^{K}(s)-K^{\gamma_{3}-\gamma_{2}} \tau_{2} V_{2}^{K}(s)\right) d s+\left\langle\mathscr{R}^{K}\right\rangle_{t} .
$$


Therefore, using again (38) and Doob's inequality, we obtain

$$
\begin{array}{rl}
\mathbb{E}\left[\sup _{t \in\left[0, T \wedge T_{n}\right]} V_{2}^{K}(t)^{2}\right] \leq 8 & \mathbb{E}\left[\int_{0}^{T \wedge T_{n}} V_{2}^{K}(s)^{2} d\left\langle\mathscr{R}^{K}\right\rangle_{s}\right]+\frac{\tau_{1}^{2}}{\tau_{2} K^{\gamma_{3}-\gamma_{2}}} \mathbb{E}\left[\int_{0}^{T \wedge T_{n}} V_{1}^{K}(s)^{2} d s\right] \\
+ & \mathbb{E}\left[\left\langle\mathscr{R}^{K}\right\rangle_{T \wedge T_{n}}\right] .
\end{array}
$$

Finally, we deduce from Lemma 2, (40), (31) and (37) that for any $K, T_{n}$ tends almost surely to $+\infty$ and that for all $T \geq 0$,

$$
\lim _{K \rightarrow \infty} \mathbb{E}\left[\sup _{t \in[0, T]} V_{2}^{K}(t)^{2}\right]=0 .
$$

(ii) Let us now prove the result (ii) of Theorem 4. It has been inspired by the proof of the main result in Kang et al. (2014). Using similar convergence arguments as in Theorem 3 and (31), we firstly observe that the sequence $\left(V_{1}^{K}\right)_{K}$ converges in law in $\mathbb{D}([0, T], \mathbb{R})$ to a continuous process $V_{1}$ defined by, for all $t$,

$$
V_{1}(t)=V_{0}^{(1)}+\sqrt{\tau_{1} x_{1}} W_{1}(t)
$$

with $W_{1}$ a standard Brownian motion.

Let us recall that from (29), (30) and (35), that for all $t$,

$V_{3}^{K}(t)=V_{3}^{K}(0)+\tau_{2}\left(1+\frac{1}{K^{\gamma_{2}}}\right) \int_{0}^{t} K^{\left(1-\gamma_{3}\right) / 2}\left(Z_{2}^{K}(s)-z_{2}^{*}\right) d s-\tau_{3} \int_{0}^{t} V_{3}^{K}(s) d s+K^{\left(1-\gamma_{3}\right) / 2} \widetilde{M}_{3}^{K}(t)$

with

$\tau_{2} \int_{0}^{t} K^{\left(1-\gamma_{3}\right) / 2}\left(Z_{2}^{K}(s)-z_{2}^{*}\right) d s=K^{\left(1-\gamma_{3}\right) / 2} \frac{Z_{2}^{K}(0)-Z_{2}^{K}(t)}{K^{\gamma_{3}-\gamma_{2}}}+\int_{0}^{t} \tau_{1} V_{1}^{K}(s) d s+\mathscr{R}_{t}^{K}$.

Hence, for all $t$,

$V_{3}^{K}(t)=V_{3}^{K}(0)+\left(1+\frac{1}{K^{\gamma_{2}}}\right) \int_{0}^{t} \tau_{1} V_{1}^{K}(s) d s-\tau_{3} \int_{0}^{t} V_{3}^{K}(s) d s-\left(1+\frac{1}{K^{\gamma_{2}}}\right) V_{2}^{K}(t)+\mathscr{M}_{t}^{K}$,

where $\mathscr{M}^{K}$ is the square-integrable martingale

$$
\mathscr{M}^{K}(t)=\left(1+\frac{1}{K^{\gamma_{2}}}\right) \mathscr{R}_{t}^{K}+K^{\left(1-\gamma_{3}\right) / 2} \widetilde{M}_{3}^{K}(t) .
$$

We deduce from (23), (36), Lemma 2 and Doob's inequality, that

$$
\lim _{K \rightarrow \infty} \mathbb{E}\left[\sup _{t \in[0, T]}\left|\mathscr{M}^{K}(t)\right|^{2}\right]=0
$$

Then it turns out from Markov's inequality that the sequence $\left(\mathscr{M}^{K}\right)_{K}$ converges in probability to 0 for the uniform norm and hence $\left(\mathscr{M}^{K}\right)_{K}$ converges in law in $\mathbb{D}([0, T], \mathbb{R})$ to 0 .

Furthermore using (42), (37), (33) and the first part of the theorem, we obtain

$$
\sup _{K} \mathbb{E}\left[\sup _{t \leq T}\left|V_{3}^{K}(t)\right|\right]<\infty .
$$


We are now able to prove the tightness of the family $\left(\sup _{t \leq T}\left|V_{3}^{K}(t)\right|\right)_{K}$. Indeed, les us introduce stopping times $S, S^{\prime}$ satisfying $S \leq S^{\prime} \leq(S+\delta) \wedge T$, with $\delta>0$. Using (42), we have

$$
\begin{aligned}
\mathbb{P}\left(\left|V_{3}^{K}\left(S^{\prime}\right)-V_{3}^{K}(S)\right|>\varepsilon\right) \leq & \frac{1}{\varepsilon} \mathbb{E}\left[\left|V_{3}^{K}\left(S^{\prime}\right)-V_{3}^{K}(S)\right|\right] \\
\leq & \frac{1}{\varepsilon} \mathbb{E}\left[\left|V_{3}^{K}\left(S^{\prime}\right)-V_{3}^{K}(S)\right|^{2}\right]^{1 / 2} \\
\leq & \frac{1}{\varepsilon}\left[\delta T\left(\tau_{1}\left(1+\frac{1}{K^{\gamma} 2}\right)\left(\mathbb{E}\left[\sup _{t \leq T} V_{1}^{K}(t)^{2}\right]+1\right)+\tau_{3} \mathbb{E}\left[\sup _{t \leq T}\left|V_{3}^{K}(t)\right|\right]\right)\right. \\
& \left.\quad+4 \mathbb{E}\left[\sup _{t \leq T} \mathscr{M}^{K}(t)^{2}\right]+4 p_{2}^{D} \mathbb{E}\left[\sup _{t \leq T} V_{2}^{K}(t)^{2}\right]\right]^{1 / 2} .
\end{aligned}
$$

Then from (42), (37), (33) and the first part of the theorem, we deduce that Aldous conditions (see Joffe and Metivier (1986) and Bansaye and Meleard (2015)) are satisfied and obtain the tightness of $\left(V_{3}^{K}\right)_{K}$.

Finally, using the first part of the theorem, the convergence in law in $\mathbb{D}([0, T], \mathbb{R})$ of the processes $\mathscr{M}^{K}$ and $V_{1}^{K}$ respectively to zero and $V_{1}$ and the convergence in law of $V_{3}^{K}(0)$ to $V_{0}^{(3)}$, we obtain that the sequence $\left(V_{3}^{K}\right)_{K}$ converges in law in $\mathbb{D}([0, T], \mathbb{R})$ to the process $V_{3}$, unique solution of the following SDE, $\forall t \in[0, T]$,

$$
V_{3}(t)=V_{0}^{(3)}+\tau_{1}\left(1+\frac{1}{K^{\gamma_{2}}}\right) \int_{0}^{t} V_{1}(s) d s-\tau_{3} \int_{0}^{t} V_{3}(s) d s,
$$

where $V_{1}$ has been defined in (41). That ends the proof.

\section{Discussion}

Our model concerns a rest hematopoiesis, and then it neglects the regulation effects (which are not observable in this situation). The large fluctuation effects that we are interested in are only due to amplification of the first component randomness.

Let us visualize in the simulations below, the quantities involved in the different theorems. As in Figures 1, 2 and 3, we choose $K=2000$ cells, $\gamma_{2}=0.55, \gamma_{3}=0.8$. Hence $K^{\gamma_{2}} \sim 60$ and $K^{\gamma_{3}} \sim 400$. The others parameters are equal to 1 .

Figure 4 illustrates the results of Theorems 1 and 2. It represents on the same picture, first the dynamics of $Y_{2}^{K}$ and $y_{2}$ (defined respectively in (10) and (11)), second the dynamics of $Z_{3}^{K}$ and $z_{3}$ (defined respectively in (15) and (17)). The common initial condition of $Y^{K}$ and of $y=\left(y_{1}, y_{2}\right)$ is given by $\left(x_{1}, 0\right)$. The initial condition of $Z^{K}$ is given by $\left(x_{1}, 0,0\right)$ and the one of $\left(z_{1}, z_{3}\right)$ by $\left(x_{1}, 0\right)$. We take $x_{1}=1$. We observe first the proximity of the two dynamics and then the fluctuations effects.

Figure 5 shows the simulation of a trajectory of the process $\left(N^{K}(t), \quad t \in[0, T]\right)$ over a long period of time (of order $K^{\gamma_{3}}$ ), starting from the equilibrium values $\left(K x_{1}, K^{1+\gamma_{2}} z_{2}^{*}, K^{1+\gamma_{2}+\gamma_{3}} z_{3}^{*}\right)$ of the deterministic limit. We take $x_{1}=1$, hence $z_{2}^{*}=$ $\frac{\tau_{1} x_{1}}{\tau_{2}}=1$ and $z_{3}^{*}=\frac{\tau_{2} z_{2}^{*}}{\tau_{3}}=1$. The horizontal orange line represents this information. 

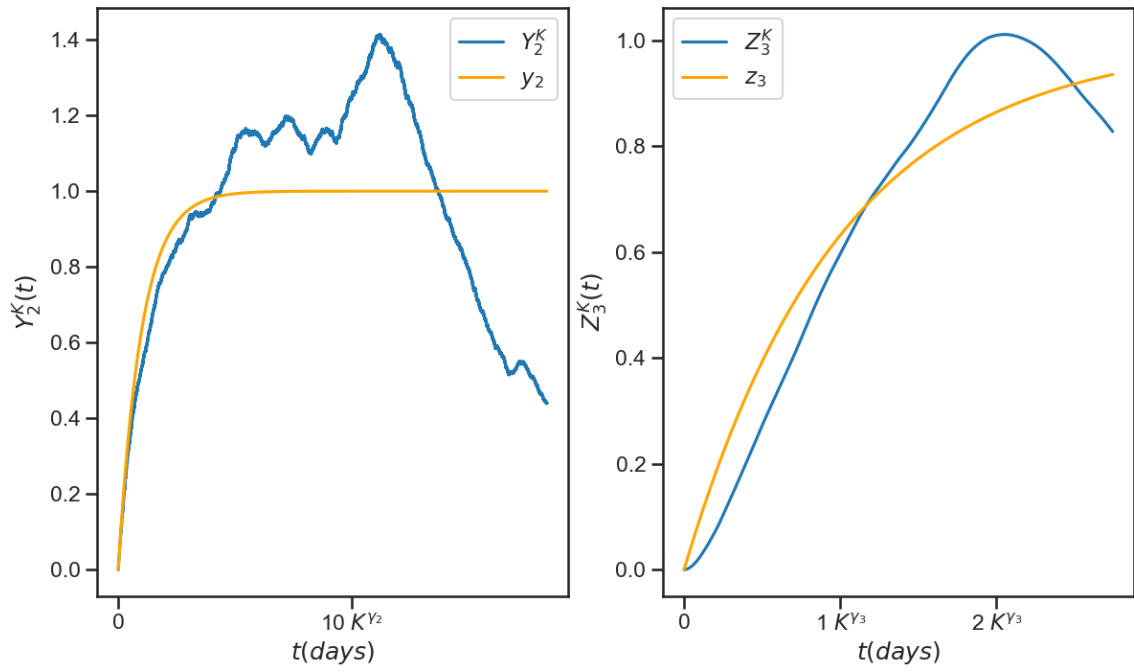

Fig. 4: Comparison of a trajectory of $Y_{2}^{K}$ with a trajectory of $y_{2}$ and of a trajectory of $Z_{3}^{K}$ with a trajectory of $z_{3}$.

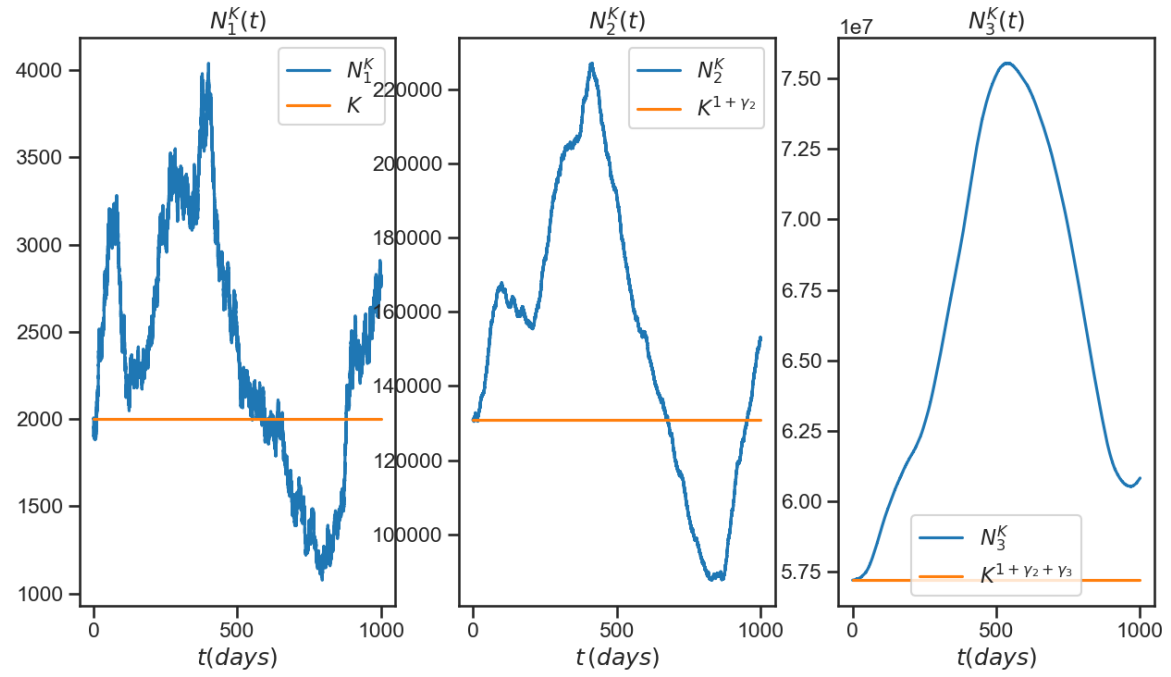

Fig. 5: A trajectory of the process $\left(N^{K}(t), \quad t \in[0, T]\right)$ for $T=1000$ (days) and starting from $\left(K, K^{1+\gamma_{2}}\right.$ $\left.K^{1+\gamma_{2}+\gamma_{3}}\right)$.

We observe on Figure 5 the two main properties of the fluctuations that we have highlighted in our results (Theorems 3 and 4): the fluctuations of the two last components are both smoother and larger than expected from standard CLT (Central Limit Theorem). Indeed following Kang et al. (2014) (and classical CLT results), the order of magnitude of the population size fluctuations should be given by the square 
of the population size, i.e., respectively $K^{\left(1+\gamma_{2}\right) / 2} \sim 400$ cells of type 2 cells and $K^{\left(1+\gamma_{2}+\gamma_{3}\right) / 2} \sim 7600$ cells of type 3 . Figure 5 shows for the second type large fluctuations of around 80000 cells and for the third type of around $1.710^{7}$ cells. Theorems 3 and 4 give these orders of magnitude, with about $K^{\left(1+3 \gamma_{2}\right) / 2} \sim 24000$ cells of type 2 and $K^{\left(1+2 \gamma_{2}+3 \gamma_{3}\right) / 2} \sim 2.710^{7}$ cells of type 3 .

As shown in Theorems 3 and 4, the augmented fluctuations observed on types 2 and 3 cells are due to the fast arrival of new cells in a slower dynamics. More precisely, the randomness of the slow component is essentially due to the fast ones and its intrinsic randomness is negligible. That is the major difference with Kang et al. (2014) where the martingale part of the limit is due to two sources of randomness: the slow component dynamics and the averaged effects of the fast component on the slow component dynamics.

Finally, let us note that a biological perspective of Theorem 4 could be to estimate the still unknown human stem cells number. Indeed, Theorem 4 estimates the variation of red blood cells number around its mean value, and (34) gives that

$$
\frac{N_{3}^{K}(t)-K^{1+\gamma_{2}+\gamma_{3}} z_{3}\left(t K^{-\gamma_{3}}\right)}{N_{3}^{K}(t)} \sim \frac{\left.K^{\left(1+2 \gamma_{2}+3 \gamma_{3}\right) / 2}\right)}{K^{1+\gamma_{2}+\gamma_{3}}}=K^{\left(\gamma_{3}-1\right) / 2} .
$$

When $K$ is (the order of magnitude of) the number of human stem cells, $K^{\gamma_{3}}$ is the number of days a human red blood cell lives (120 days). We know that the human red blood cells number at rest varies by $10 \%$ around its average value (cf. Thirup (2003)). Hence we can deduce that the order of magnitude of the human stem cells number is $K=\frac{120}{0.01} \sim 12000$. In Abkowitz et al. (2002), it is shown that the number of human stem cells is equivalent to the one of mice. For mice, the estimated number of stem cells is of order of magnitude $10^{4}$ (cf Busch et al. (2015), Bonnet et al. (2021)). That corroborates our result.

\section{Appendix}

Lemma 3 (Lemma 2.9 of Kang et al. (2014)) Let $V^{N}$ be a sequence of $\mathbb{R}_{+}^{3}$-valued processes. We consider its occupation measure defined for $D$ a Borelian set by

$$
\Gamma_{N}(D \times[0, t])=\int_{0}^{t} \mathbf{1}_{\mathbf{D}}\left(\mathbf{V}^{\mathbf{N}}(\mathbf{s})\right) \mathbf{d s} .
$$

Let us assume that there exists a function $\psi: \mathbb{R}_{+}^{3} \rightarrow[1, \infty)$ locally bounded such that $\lim _{v \rightarrow+\infty} \psi(v)=+\infty$ and such that for each $t>0$,

$$
\sup _{N} \mathbb{E}\left[\int_{0}^{t} \psi\left(V^{N}(s)\right) d s\right]<+\infty .
$$

Then $\Gamma_{N}$ is relatively compact, and if $\Gamma_{N}$ converges in law to $\Gamma$, then for $f_{1}, \ldots, f_{m} \in$ $D_{\psi}$,

$$
\begin{aligned}
\left(\int_{0} f_{1}\left(V_{N}(s)\right) d s, \ldots, \int_{0} f_{m}\left(V^{N}(s)\right) d s\right) \stackrel{\mathscr{L}}{\longrightarrow} \\
\left(\int_{\mathbb{R}_{+}^{3}} f_{1}(v) \Gamma(d v \times[0, .]), \ldots, \int_{\mathbb{R}_{+}^{3}} f_{m}(v) \Gamma(d v \times[0, .])\right)
\end{aligned}
$$


where $D_{\psi}$ denote the collection of continuous functions $f$ satisfying

$$
\sup _{v \in \mathbb{R}_{+}^{3}} \frac{|f(v)|}{\psi(v)}<\infty \quad \text { and } \lim _{k \rightarrow \infty} \sup _{v \in \mathbb{R}_{+}^{3},\|v\|>k} \frac{|f(v)|}{\psi(v)}=0 .
$$

Acknowledgements We warmly thank Vincent Bansaye, the hematologist Stéphane Giraudier and the biologist Evelyne Lauret for exciting and fruitful discussions which have motivated this work. We also thank Vincent Bansaye for his precious comments on our paper. This work was supported by a grant from Région Île-de-France.

\section{References}

Abkowitz J, Catlin S, McCallie M, PGuttorp (2002) Evidence that the number of hematopoietic stem cells per animal is conserved in mammals. Blood 100(7):2665-2667, DOI https://doi.org/10.1182/blood-2002-03-0822, URL https://www.sciencedirect.com/science/article/pii/S0006497120542146

Abkowitz JL, Golinelli D, Harrison DE, Guttorp P (2000) In vivo kinetics of murine hemopoietic stem cells. Blood 96(10):3399-3405

Arino O, Kimmel M (1986) Stability analysis of models of cell production systems. Mathematical Modelling 7(9-12):1269-1300

Axelrod D, Kimmel M (2002) Branching Processes in Biology. Springer, New York

Bansaye V, Meleard S (2015) Stochastic Models for Structured Populations: Scaling Limits and Long Time Behavior. Mathematical Biosciences Institute Lecture Series, Springer International Publishing

Bonnet C, Gou P, Girel S, Bansaye V, Lacout C, Bailly K, Schlagetter MH, Lauret E, Meleard S, Giraudier S (2021) Combined biological and modeling approach of hematopoiesis: From native to stressed erythropoiesis. Available at SSRN: https://ssrncom/abstract=3777468 or http://dxdoiorg/102139/ssrn3777468

Busch K, Klapproth K, Barile M, Flossdorf M, Holland-Letz T, Schlenner S, Reth M, Höfer T, Rodewald HR (2015) Fundamental properties of unperturbed haematopoiesis from stem cells in vivo. Nature 18(518):542-546, DOI 10.1038/nature 14242

Crauste F, Pujo-Menjouet L, Génieys S, Molina C, Gandrillon O (2008) Adding selfrenewal in committed erythroid progenitors improves the biological relevance of a mathematical model of erythropoiesis. Journal of theoretical biology 250(2):322338

Dingli D, Traulsen A, Pacheco J (2007) Compartmental architecture and dynamics of hematopoiesis. PlosOne

Domen J, Cheshier SH, Weissman IL (2000) The Role of Apoptosis in the Regulation of Hematopoietic Stem Cells: Overexpression of BCL2 Increases Both Their Number and Repopulation Potential. Journal of Experimental Medicine 191(2):253-264, DOI 10.1084/jem.191.2.253, URL https://doi.org/10.1084/jem.191.2.253, https://rupress.org/jem/articlepdf/191/2/253/1124951/99-1505.pdf 
Ethier S, Kurtz T (1986) Markov processes - characterization and convergence. Wiley Series in Probability and Mathematical Statistics: Probability and Mathematical Statistics, John Wiley \& Sons Inc., New York

Gonzalez M, Puerto IM, Martinez R, Molina M, Mota M, Ramos A (2010) Workshop on branching processes and their applications, vol 197. Springer Science \& Business Media

Ikeda N, Watanabe S (1989) Stochastic Differential Equations and Diffusion Processes, 2nd ed. North-Holland

Joffe A, Metivier M (1986) Weak convergence of sequences of semimartingales with applications to multitype branching processes. Advances in Applied Probability 18(1):20-65

Kang HW, Kurtz TG, Popovic L (2014) Central limit theorems and diffusion approximations for multiscale markov chain models. The Annals of Applied Probability 24(2):721-759

Khammash M, Munsky B, Peleš S (2006) Reduction and solution of the chemical master equation using time scale separation and finite state projection. The Journal of chemical physics 125(20)

Kimmel M, Wazewska-Czyzewska M (1982) Stochastic approach to the process of red cell destruction. Applicationes Mathematicae 2(17):217-225

Kurtz T (1992) Averaging for martingale problems and stochastic approximation. In: Applied Stochastic Analysis, Springer, pp 186-209

Lei J, Mackey MC (2007) Stochastic differential delay equation, moment stability, and application to hematopoietic stem cell regulation system. SIAM journal on applied mathematics 67(2):387-407

Loeffler M, Wichmann H (1980) A comprehensive mathematical model of stem cell proliferation which reproduces most of the published experimental results. Cell Proliferation 13(5):543-561

Marciniak-Czochra A, Stiehl T, Ho AD, Jäger W, Wagner W (2009) Modeling of asymmetric cell division in hematopoietic stem cells-regulation of self-renewal is essential for efficient repopulation. Stem cells and development 18(3):377-386

Pazdziorek PR (2014) Mathematical model of stem cell differentiation and tissue regeneration with stochastic noise. Bulletin of mathematical biology 76(7):16421669

Roeder I, Loeffler M (2002) A novel dynamic model of hematopoietic stem cell organization based on the concept of within-tissue plasticity. Experimental hematology 30(8):853-861

Thirup P (2003) Haematocrit. Sports Medicine 33(3):231-243

Till JE, McCulloch EA, Siminovitch L (1964) A stochastic model of stem cell proliferation, based on the growth of spleen colony-forming cells. Proceedings of the National Academy of Sciences of the United States of America 51(1):29

Whichard ZL, Sarkar CA, Kimmel M, Corey SJ (2010) Hematopoiesis and its disorders: a systems biology approach. Blood, The Journal of the American Society of Hematology 115(12):2339-2347 